This item was submitted to Loughborough's Research Repository by the author.

Items in Figshare are protected by copyright, with all rights reserved, unless otherwise indicated.

\title{
Fuzzy logic control for use in in-pipe mobile robotic system navigation
}

PLEASE CITE THE PUBLISHED VERSION

PUBLISHER

Professional Engineering Publishing / @ IMECHE

VERSION

VoR (Version of Record)

LICENCE

CC BY-NC-ND 4.0

REPOSITORY RECORD

Ong, J.K., Kaddour Bouazza-Marouf, and David Kerr. 2019. "Fuzzy Logic Control for Use in In-pipe Mobile Robotic System Navigation”. figshare. https://hdl.handle.net/2134/5146. 
This item was submitted to Loughborough's Institutional Repository (https://dspace.lboro.ac.uk/) by the author and is made available under the following Creative Commons Licence conditions.

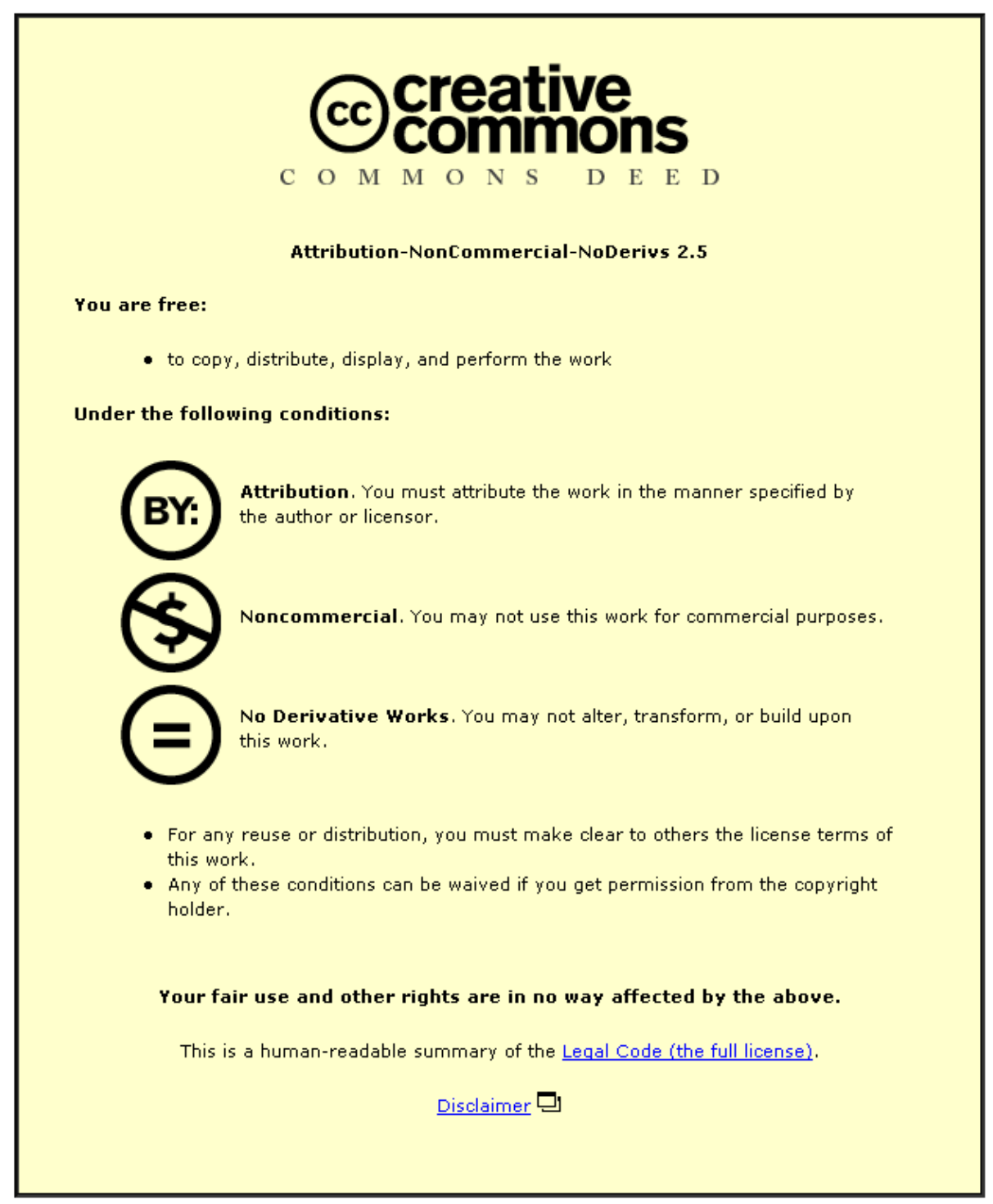

For the full text of this licence, please go to: http://creativecommons.org/licenses/by-nc-nd/2.5/ 


\title{
Fuzzy logic control for use in in-pipe mobile robotic system navigation
}

\author{
J K Ong*, K Bouazza-Marouf and D Kerr \\ Wolfson School of Mechanical and Manufacturing Engineering, Loughborough University, Leicestershire, UK
}

\begin{abstract}
This paper presents a fuzzy logic control for the navigation of a mobile robotic system in gas pipelines. The robotic system is designed for a local gas distribution pipeline network with $150-300 \mathrm{~mm}$ diameter pipes; common pipe fittings in use are straight and bend sections, reducers and slope pipe sections. The navigation problem forms a part of the current development of a new modular and semi-autonomous vehicle system. The vehicle control and navigation technique is implemented using a two-mode controller consisting of a proportional-integral-derivative (PID) and fuzzy logic control. The PID controller is responsible for direct control of the actuators, while the fuzzy logic controller is used to evaluate as well as to define the sensor outputs such as speed, climbing angle and rate of climbing angle in order to perceive the different types of pipe environment and vehicle actions. Since the navigation problem involves a multivariable input-output (MIMO) system, a cascaded hierarchical fuzzy model configuration is used to reduce the dimensionality of the fuzzy model. The fuzzy navigation controller is thus an interlink fuzzy subsystem of the pipe environment recognition and action adjustment subsystems. Results of simulations and laboratory experiments are presented to demonstrate the ability of the control strategy. A brief description of the mobile robotic system used is presented as background.
\end{abstract}

Keywords: gas pipe operation tool, robotic system, navigation control in gas pipes, fuzzy logic controller

\section{NOTATION}

$A_{\mathrm{v}} \quad$ action control signal

$d \quad$ distance between wheels

$K \quad$ number of fuzzy variables (quantity)

$n \quad$ number of fuzzy output sets

(granularity)

$R_{i j} \quad$ fuzzy linguistic rule, $i, j=1,2,3, \ldots, n$

$\Delta S_{\mathrm{L}} \quad$ left wheel travel distance

$\Delta S_{\mathrm{R}} \quad$ right wheel travel distance

$\Delta T \quad$ time step

$\Delta V \quad$ speed difference

$V_{\mathrm{D}} \quad$ desired driving speed

$V_{\mathrm{L}} \quad$ left wheel speed

$V_{\mathrm{LC}} \quad$ corrected left wheel speed

$V_{\mathrm{R}} \quad$ right wheel speed

$V_{\mathrm{RC}} \quad$ corrected right wheel speed

$w_{i} \quad$ degree of membership of the $i$ th fuzzy rule

The MS was received on 25 February 2003 and was accepted after revision for publication on 28 April 2003.

* Corresponding author: Wolfson School of Mechanical and Manufacturing Engineering, Loughborough University, Loughborough, Leicestershire LE11 3TU, UK $\bar{x}_{1}, \bar{x}_{2}, \bar{x}_{3}$

$\bar{z}_{1}, \bar{z}_{2}$

$\theta$

$\theta_{\mathrm{D}}$

$\varepsilon_{\mathrm{L}}, \varepsilon_{\mathrm{R}}$

fuzzy elements of the input variables singleton outputs from the fuzzy system

climbing angle

desired climbing angle

surface slip factor

\section{INTRODUCTION}

Industries that operate gas, water, oil or chemical pipeline networks spend large sums of money on pipe maintenance operations. Many tools such as pipe pigs or push-rod-type camera systems exist, and the use of the tools is determined by factors such as cost, safety and pipe environment as well as type of services. In many situations, mobile robotic systems have gained an edge over the conventional tools. A report prepared for the Gas Research Institute (GRI) workshop on robotic applications for gas pipe operators has identified research aspects including new versatile robotic systems, issues on powering such systems, autonomous versus teleoperation control, data and control signal communication, propulsion, etc. [1]. 
Currently, in-pipe locomotion seems to be the major focus with research work carried out by researchers in this field [2-6]. Most of the authors describe various mechanical designs involving wheel-driven configurations and legged, inch-worm and crawler mechanisms. The developed robotic systems are claimed to be able to overcome a certain range and type of pipe environment. They are all remotely controlled by an operator. A logical development of current work would lead to the ability of self-navigation. This would offer advantages such as time saving and operation efficiency.

For humans, the ability to navigate is eminent. For mobile robotic systems, however, navigation in a realworld environment is a complex and challenging task. Generally speaking, a robot has to make decisions about the actions it should take in each time-step, considering the information obtained from its sensors about the environment state. This requires processing of sensory inputs to provide appropriate outputs according to how the operator wants the robotic system to behave. To perform this task, many control techniques can be used, such as linear transfer functions, expert systems, neural networks, fuzzy systems and others [7-11].

The goal of this research is to find a controller technique that, given the parameters of the robotic system and sensory data, delivers suitable actions for the robot to navigate within the pipe environments. At the moment, as far as is known, no such control strategy for in-pipe robotic systems exists. Nevertheless, open space mobile robot navigation strategies are widely available and present related insights for constrained in-pipe environment navigation. One of the commonly used techniques for robot navigation is Landmark Navigation, in which the robot recognizes the environment and orientates towards it [12-14]. This method is known to have a trade-off between computation complexity and execution time [15]. Another possible navigation technique is Navigation by Map [16, 17]. However, the method may not be suitable as it is hard to obtain a proper and informative pipeline construction layout for many existing installations.

The approach adopted here is to have the behaviour of the robotic vehicle expressed in fuzzy rules and to apply these rules continually in order to produce an appropriate reaction in the face of a changing environment. By adopting such a strategy, the operator only needs to specify the desired settings of the robotic system. A set of linguistic rules is used to guide the vehicle through different pipe fittings, performing necessary adjustment to its states.

This paper addresses the issues of pipe fittings recognition and navigation control for an in-pipe mobile robotic system by a fuzzy logic technique. The fuzzy logic controller developed for this system consists of a hybrid of cascaded and hierarchical evaluation of fuzzy rule sets to carry out two stages of a navigation inference process. For clarity, the paper also briefly describes the mobile robotic system [18] in section 2.1 and the navigational issues in section 2.2. The proposed navigation strategy and the design of the navigational controller are given in sections 3 and 4. Both simulation and experimental test results showing the ability of the fuzzy controller are presented in sections 5 and 6 .

\section{IN-PIPE ROBOTIC SYSTEM}

\subsection{The robotic vehicle}

Currently available in-pipe robotic systems are teleoperated, which therefore represents, at best, fairly simple control techniques [19]. Hence, these systems are built with complicated mechanisms to allow motions within the pipe environment. In contrast, the authors have developed a simple differential drive wheeled-type robotic vehicle $[\mathbf{1 8}, \mathbf{2 0}]$ to demonstrate an intelligent control approach to in-pipe locomotion. The robotic system consists of a cylindrical chassis with two independently powered wheel assemblies mounted on each side of a common axis. This enables the robotic vehicle to drive straight ahead (i.e. no speed difference between the two sides), curve or turn (i.e. with speed difference). The schematics of the in-pipe robotic vehicle are shown in Fig. 1. The vehicle dimensions are $152 \mathrm{~mm}$ in length and $114 \mathrm{~mm}$ in diameter. The robotic vehicle has integrated optical encoders and a tilt sensor to offer real time information about the states of the system as well as the abstraction of the pipe environment.

\subsection{Navigation issues}

The use of the simplistic differential drive system produces an area of concern. Since the wheels, motors and gearing systems may not match, vehicle movement may result in a veering action to either the left or right. Calibrating the motors and wheels to rotate at the same speed may not necessarily solve the problem as the wheels themselves may encounter different surfaces and loads. Controlling the individual velocity of the wheels dynamically, i.e. monitoring and altering the speeds of the wheels, must be done while the vehicle is driving. This form of control can be easily implemented using a proportional-integral-derivative (PID) controller, forming a low-level part of the navigation control strategy. However, the environment must be identified to allow accurate vehicle speed control. As such, the robotic vehicle is required to employ higher level, intelligent reactive type sensors and control strategies.

There are three recognized problems in motion control of mobile robotic systems in pipelines [21]. Firstly, the underground pipeline environment is not completely known. Secondly, this environment is only partially observable. Finally, the action of the robotic system may not be executed correctly. It is clear that a robotic vehicle 


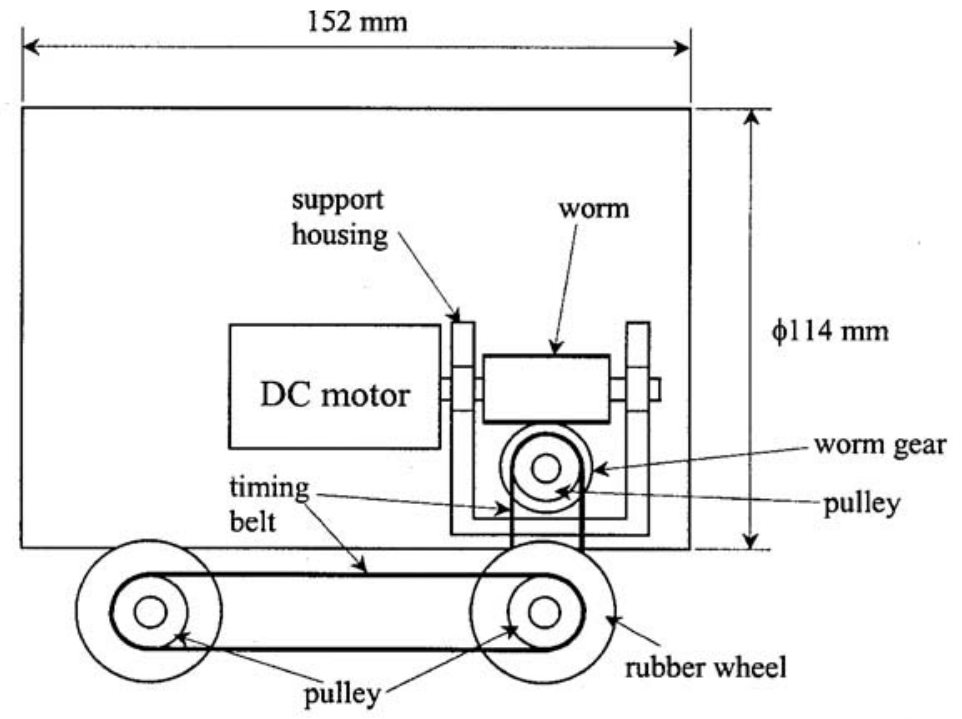

(a) The driving mechanism (scale 1:2) of the in-pipe robotic vehicle

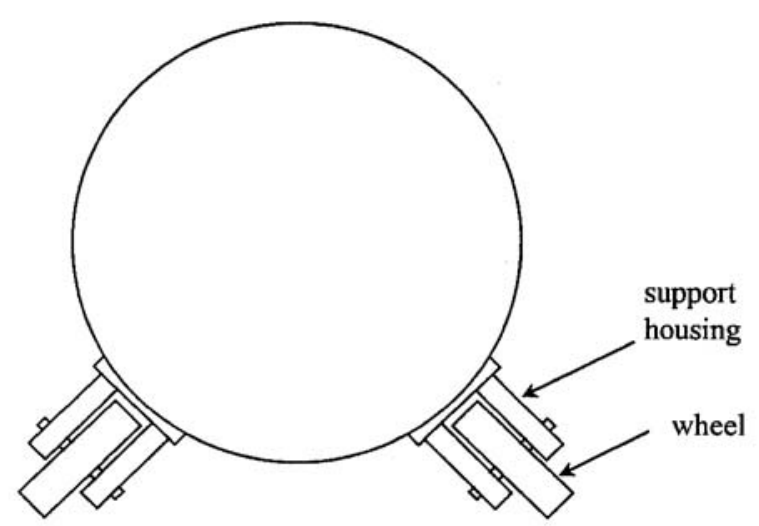

(b) The arrangement of the wheels

Fig. 1 Schematics of the robotic vehicle unit

in gas pipelines faces environment variability and uncertainty. In this particular research context, these factors indicate that the design of the control technique has to be carefully selected and planned to handle the wide variety of encounters and problems in the pipeline environment.

As a whole, the complexity of the control mechanism is traded off due to the complexity of the pipe environment and the simplicity of the robotic system used. Nonetheless, the end result should prove to be a reliable and cost effective trade-off.

\section{CONTROL STRATEGY}

The capability of representing uncertain or imprecise knowledge about an environment as well as motion planning for the robotic system in the environment are very important for any self-navigating robotic system. A set of rules that define the robot's actions given the environmental constraints is one of many valid approaches. Fuzzy logic is able to perform different levels of abstraction and interpretation of sensor information, based on sets of rules and defuzzification calculations, and to output control signals to the robotic system. Thus, the navigation control strategy for this self-navigation in-pipe robotic system is focused on:

(a) motion control that guarantees robust navigation in spite of a limited knowledge of the environment, e.g. allows climbing to avoid pipe take-offs and obstacles;

(b) recognition of several possible pipe fittings in order to allow the robotic vehicle to carry out smooth movement;

(c) coordinating the actions of the robotic vehicle when certain pipe fittings are encountered.

The controller developed in this research is focused in

Proc. Instn Mech. Engrs Vol. 217 Part I: J. Systems and Control Engineering 
its scope in order to provide an effective vehicle motion control in the gas pipeline environment, which is characterized by specific constraints. The navigation controller scheme is shown in Fig. 2.

In Fig. 2, the PID controller is used for controlling the vehicle speed and climbing angle, whereas the fuzzy logic controller is included in the overall loop as a supervisory controller. A Takagi-Sugeno-type fuzzy inference system [22] is used to develop the fuzzy logic algorithm. The main roles of the fuzzy logic algorithm are to recognize types of pipe fittings, measure vehicle actions and decide whether further corrective action is needed. The corrective action, provided by the 'action control signal $A_{\mathrm{v}}$ ', is necessary when the vehicle encounters pipe fittings and when there is undesired drift. The right and left speeds and climb angle are therefore adjusted to negotiate pipe fittings and also avoid drift. The fuzzy logic navigation controller is discussed below.

\section{NAVIGATION CONTROLLER}

Execution of the navigation controller is implemented such that:

1. Any demand on speed and climbing angle input by the operator should be met.

2. The pipe fittings recognition algorithm should always be active but should not affect the normal operation of the vehicle navigation controller.

The first criterion can be achieved by a PID control algorithm. The second criterion requires high-level fuzzy logic navigation control and pipe fittings recognition to be integrated in order to prevent conflicts. Therefore, as a requirement, the control architecture should support parallel execution of several low-level programmed processes [e.g. pulse width modulation (PWM) regulation or sensor sampling] and high-level tasks (e.g. navigation or optimization of actions).
The robotic vehicle motion can be measured and is directly related to the driving speed, $V$, obtained from the vehicle left and right speeds $\left(V_{\mathrm{R}}\right.$ and $\left.V_{\mathrm{L}}\right)$, and its climb angle, $\theta$. The three states are referenced to the initial operator commands, i.e. operator desired speed $\left(V_{\mathrm{D}}=V_{\mathrm{RD}}=V_{\mathrm{LD}}\right)$ and climb angle $\left(\theta_{\mathrm{D}}\right)$, to measure any deviations from the desired motion. The climb angle is measured relative to the vertical axis. A further state variable involves the vehicle climb angle rate $\mathrm{d} \theta / \mathrm{d} t$, which provides further information on pipe environment, particularly different types of bend sections as the robotic vehicle is driving through them.

Referring to Fig. 3, which represents a simplified planar motion of the vehicle system, a velocity difference, $\Delta V$ (i.e. $\Delta V=V_{\mathrm{R}}-V_{\mathrm{L}}$ ), causes an unequal travel distance between the driving wheels. The following equations describe the motion in straight pipes:

$$
\begin{aligned}
& \Delta S_{\mathrm{L}} \approx V_{\mathrm{L}}\left(1-\varepsilon_{\mathrm{L}}\right) \Delta T \\
& \Delta S_{\mathrm{R}} \approx V_{\mathrm{R}}\left(1-\varepsilon_{\mathrm{R}}\right) \Delta T
\end{aligned}
$$

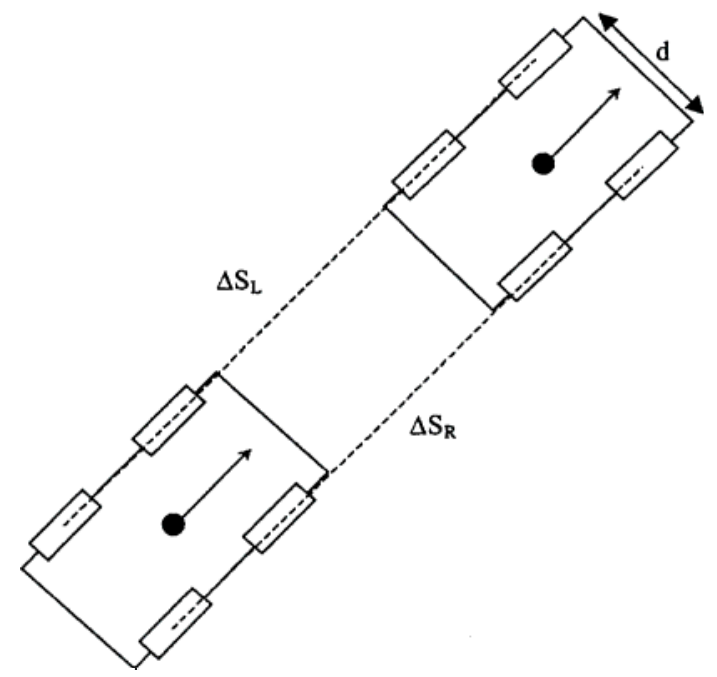

Fig. 3 Simplified planar motion of the in-pipe robotic system

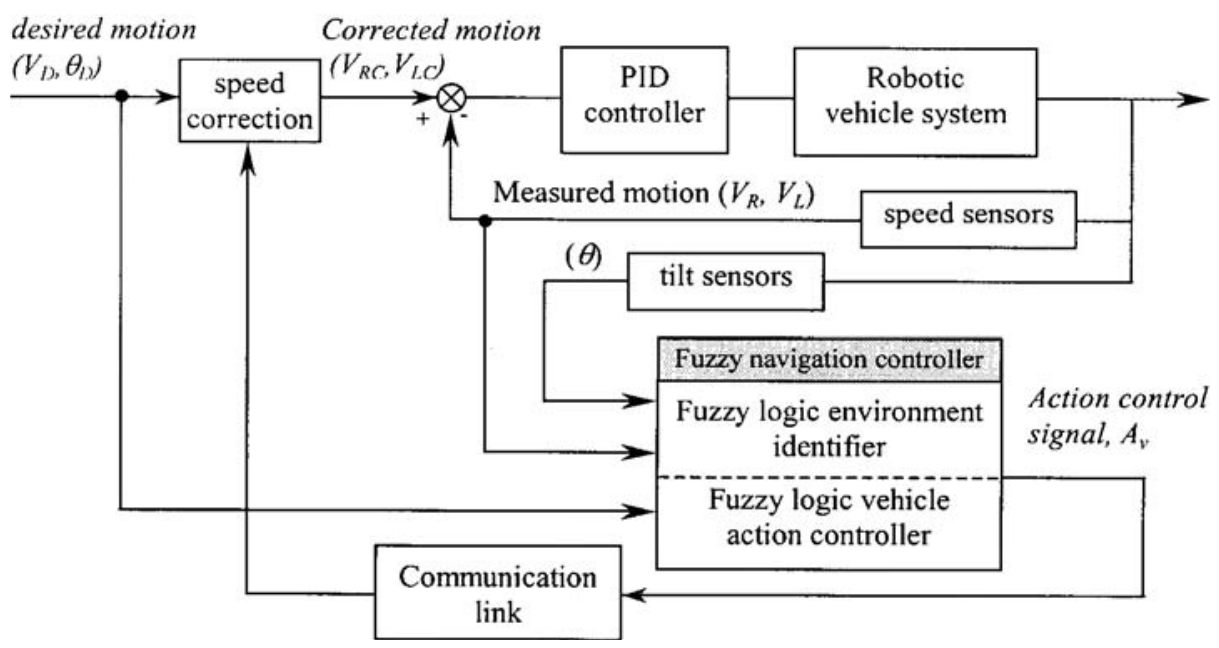

Fig. 2 The navigation controller scheme 
Instead of causing a heading direction error (i.e. turning left or right), such as in open space navigation, the difference in the distance travelled by the left and right sides, given by $\Delta S_{\mathrm{L}}$ and $\Delta S_{\mathrm{R}}$, will cause the robotic vehicle to climb up the pipe wall. This will result in the climb angle $\theta \neq 0$. However, it is necessary for the left and right speeds of the vehicle to be different in order to negotiate pipe bends. This will result in the vehicle climbing the pipe wall around the bend. The climb angle is a function of the bend geometry and the size of the vehicle. Such characteristics of the robotic vehicle drive and pipe environment are important in forming the basis of the fuzzy navigation technique. The fuzzy navigation needs, firstly, to deduce the pipe environment from sensory data and, secondly, to apply corrections $\left(A_{\mathrm{v}}\right)$ to current vehicle actions.

\subsection{Fuzzy rules reduction}

The vehicle fuzzy navigation system is a multivariable input-output (MIMO) system, with three state inputs and two outputs. A well-known problem in fuzzy logic is that the size of the fuzzy controller depends heavily on the quantity (number of variables) and granularity (number of membership functions per variable) of the system [23]. The expressive power of fuzzy logic decreases with the increase in the rules dimension. In earlier work [24-26] rule reduction in fuzzy systems has been attempted via genetic algorithms, neural networks and a variety of clustering techniques. These techniques can learn to select only those rules that contribute the most to the inference outcome. Also, another approach is to use the disjunctive form of the conjunctive rule in the if-then rules [27].

A less complicated technique, using a cascaded hierarchical fuzzy system configuration, is introduced here to reduce the rules dimensionality for this navigation application. The configuration is constructed by interconnecting multivariable input-single output (MISO) fuzzy subsystems in a hierarchical and cascaded structure. In such a case, an output of one MISO subsystem becomes an input to the subsequent MISO subsystem, such as depicted in Fig. 4. The fuzzy model now has two second-order subsystems rather than being a third-order model. The MIMO system is conveniently written as a logical combination of several MISO subsystems for easy processing. In addition, this configuration clearly shows the strong relation between the inferred pipe environ-

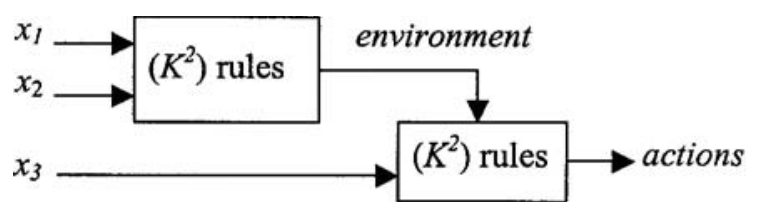

Fig. 4 Cascaded hierarchical fuzzy system configuration ment and vehicle actions, as can be observed from the simulation and experiment results in sections 5 and 6 . This approach takes the general form:

$$
\begin{aligned}
& \mathscr{R}_{i}: \text { If } X_{1} \text { is } \bar{x}_{1} \text { and } X_{2} \text { is } \bar{x}_{2} \text {, then environment is } \bar{z}_{1} \\
& \ldots \\
& \Re_{j}: \text { If environment is } \bar{z}_{1} \text { and } X_{3} \text { is } \bar{x}_{3} \text {, then action is } \bar{z}_{2} \\
& \ldots \\
& \text { where } \\
& \quad X_{1}, X_{2}, X_{3}=\text { input variables (e.g. } X_{1}=\Delta V \text { ) } \\
& \quad \bar{x}_{1}, \bar{x}_{2}, \bar{x}_{3}=\text { fuzzy elements of input variables } \\
& \quad X_{1}, X_{2}, X_{3} \\
& \quad \bar{z}_{1}, \bar{z}_{2}=\text { fuzzy rule consequents }
\end{aligned}
$$

\subsection{Pipe fittings recognition fuzzy identifier}

The pipe fittings recognition identifier is responsible for interpreting optical encoder and tilt sensor data, in terms of $\Delta V, \theta$ and $\mathrm{d} \theta / \mathrm{d} t$, into types of pipe fittings the vehicle is moving in. Initially, simpler fuzzy membership function sets for each variable were used, consisting of 9 membership functions for both the measured speed difference and angle rate and 11 membership functions for the desired climb angle. The quantization of the membership functions is based on the analysis of the motion equations and sensor data obtained empirically. Although these initial membership function sets produced an acceptable abstraction of the pipe environment, there were a few inadequacies. For example, the initial fuzzy membership functions did not provide accurate differentiation on the pipe environment when the vehicle was driven at a slower speed. In order to provide a better pipe fittings recognition ability at slower vehicle speed, as well as fuzzy validation for a wider range of input variable values, a set of 19 membership functions has been used. These membership functions are grouped closer together around the zero climb angle rate to provide closer detection of small angle rates when the vehicle is driven at a slower speed within bend sections. Improved control can also be achieved by narrowing the range of each membership function and providing more membership functions in the measured speed difference and desired angle. As a result of further tuning, the final membership functions are: 13 membership functions for the measured speed difference, 11 membership functions for the desired angle and 19 membership functions for the angle rate. The final fuzzy input membership function sets for the controller are shown in Fig. 5 and their respective linguistic descriptions are given in Table 1. The linguistic descriptions take words/sentences as values so that they can be used in forming fuzzy rules.

The outputs of the inference results are crisp values representing the pipe environment types, as shown in Fig. 6. The interaction between the input-output variables is expressed as fuzzy if-then rules. Eventually, the defuzzification process maps the fuzzy outputs into non-fuzzy values. A commonly used defuzzification 


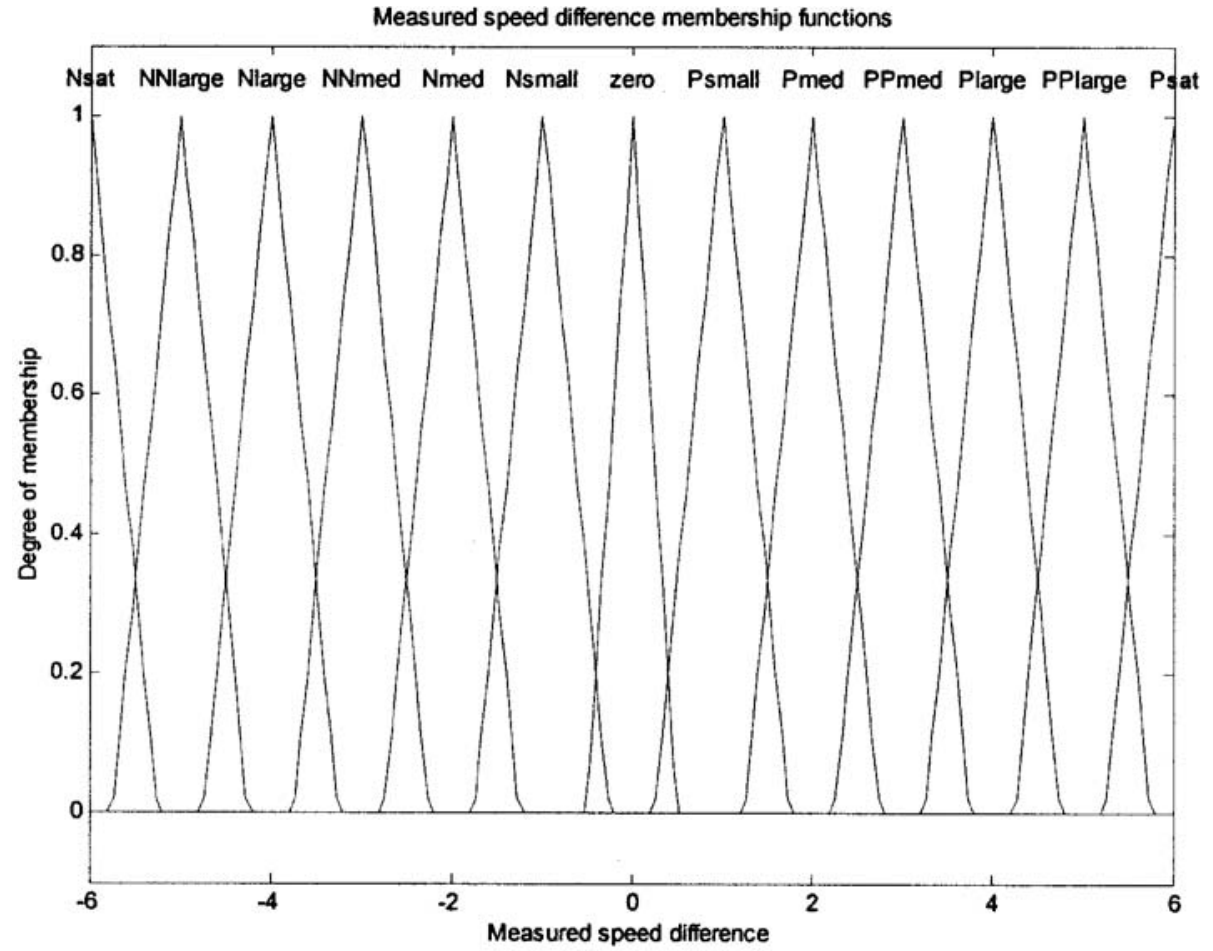

(a) Measured speed difference (level)

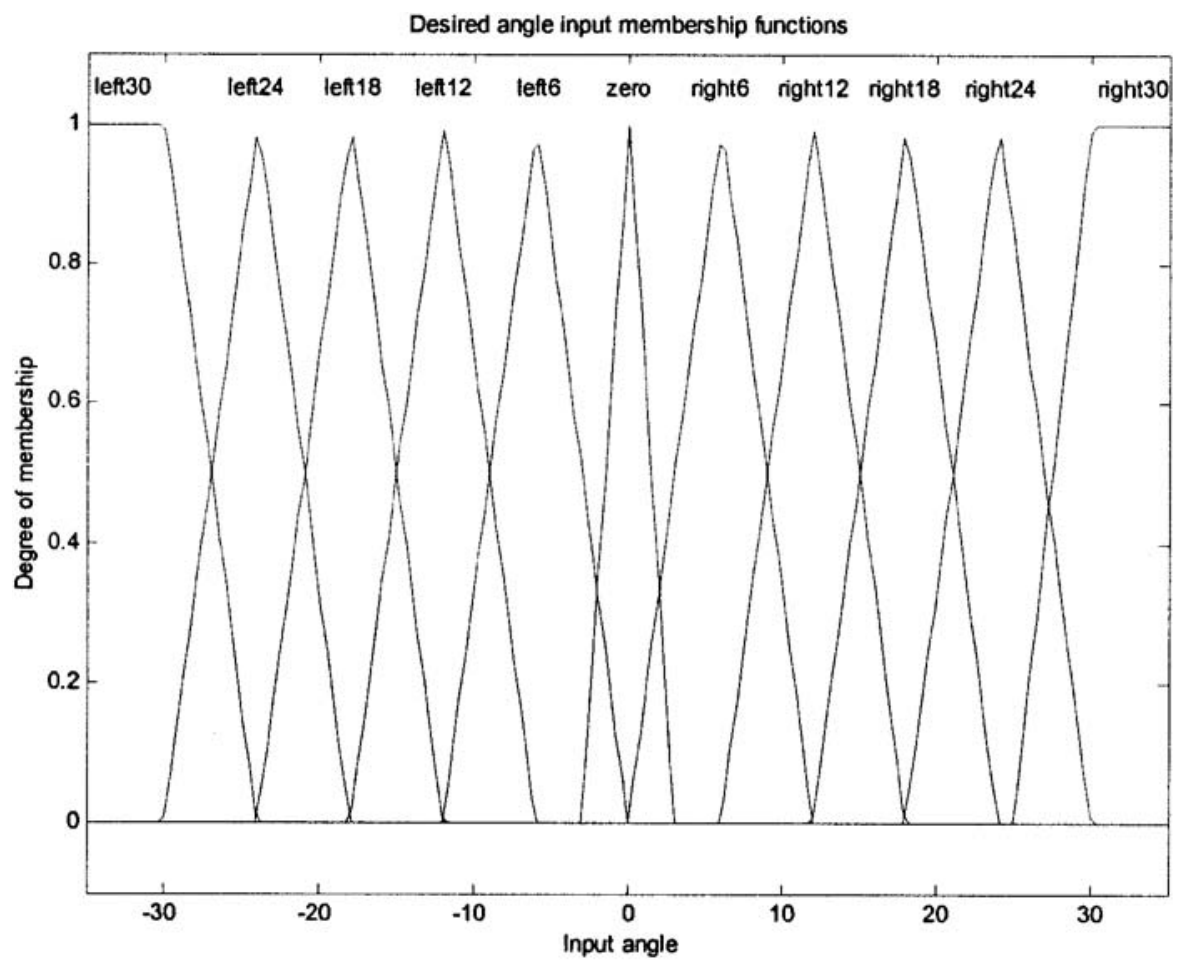

(b) Input angle (deg)

Fig. 5 (Continued over) 


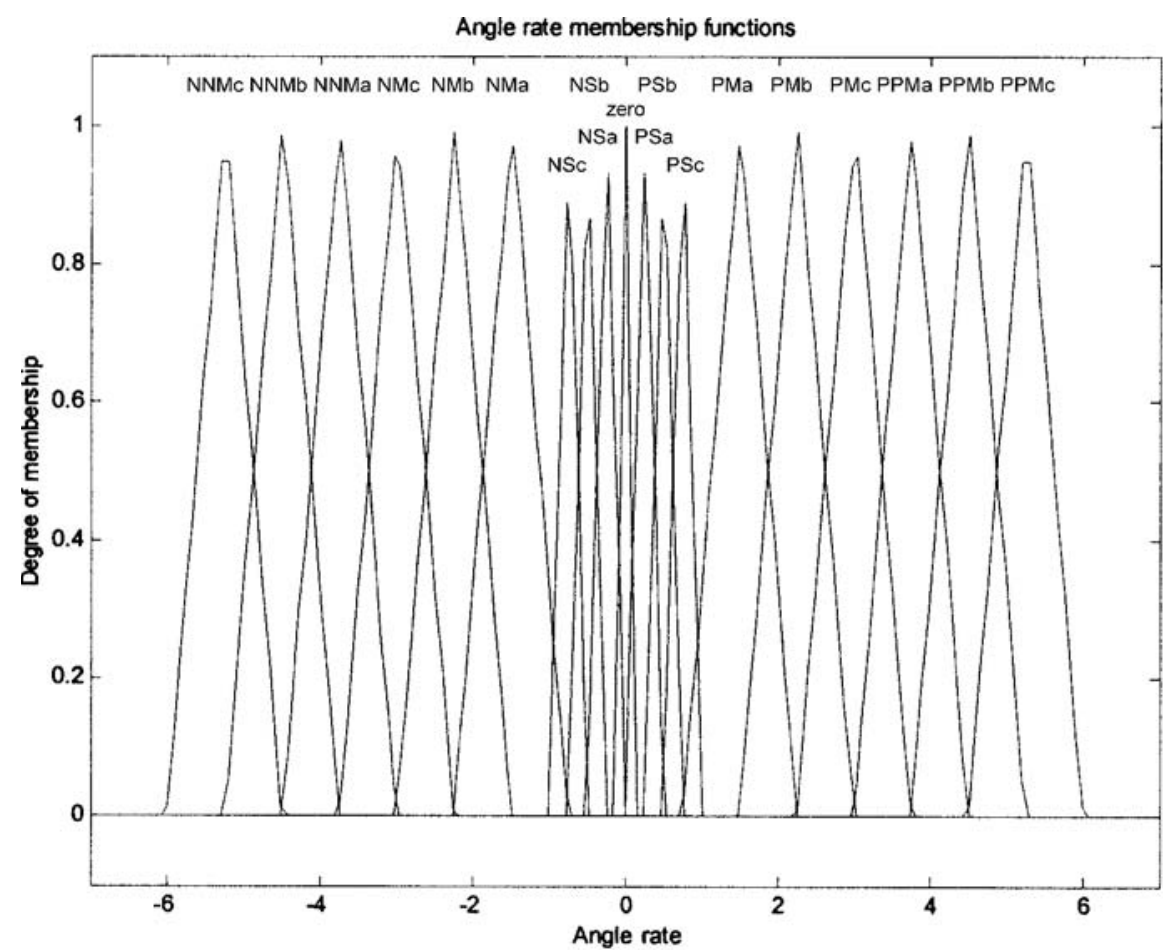

(c) Angle rate $(\mathrm{deg} / \mathrm{sec})$

Fig. 5 Input variable membership function sets for the pipe fittings recognition fuzzy controller

Table 1 Membership function linguistic descriptions of (a) speed difference, (b) desired angle and (c) angle rate input variables

\begin{tabular}{|c|c|c|c|}
\hline Label & Description & Label & Description \\
\hline \multicolumn{4}{|c|}{ (a) Measured speed difference membership set } \\
\hline Nsat & Negatively saturated & Psmall & Positively small \\
\hline NNlarge & Very negatively large & Pmed & Positively medium \\
\hline NLarge & Negatively large & PPmed & Very positively medium \\
\hline NNmed & Very negatively medium & Plarge & Positively large \\
\hline Nmed & Negatively medium & PPlarge & Very positively large \\
\hline Nsmall & Negatively small & Psat & Positively saturated \\
\hline Zero & Zero & & \\
\hline \multicolumn{4}{|c|}{ (b) Desired angle membership set } \\
\hline Left 30 & Around left $30^{\circ}$ & Right6 & Around right $30^{\circ}$ \\
\hline Left 24 & Around left $24^{\circ}$ & Right12 & Around right $30^{\circ}$ \\
\hline Left 18 & Around left $18^{\circ}$ & Right18 & Around right $30^{\circ}$ \\
\hline Left12 & Around left $12^{\circ}$ & Right24 & Around right $30^{\circ}$ \\
\hline Left6 & Around left $6^{\circ}$ & Right30 & Around right $30^{\circ}$ \\
\hline Zero & Zero angle & & \\
\hline \multicolumn{4}{|c|}{ (c) Angle rate membership set } \\
\hline NNMc & Very negatively large & Zero & Zero angle rate \\
\hline $\mathrm{NNMb}$ & Somewhat negatively large & $\mathrm{PSa}$ & Very positively small \\
\hline NNMa & Negatively large & $\mathrm{PSb}$ & Somewhat positively small \\
\hline NMc & Very negatively medium & PSc & Positively small \\
\hline $\mathrm{NMb}$ & Somewhat negatively medium & PMa & Positively medium \\
\hline $\mathrm{NMa}$ & Negatively medium & $\mathrm{PMb}$ & Somewhat positively medium \\
\hline $\mathrm{NSc}$ & Negatively small & PMc & Large positively medium \\
\hline $\mathrm{NSb}$ & Somewhat negatively small & PPMa & Positively large \\
\hline \multirow[t]{2}{*}{$\mathrm{NSa}$} & Very small & PPMb & Somewhat positively large \\
\hline & & PPMc & Very positively large \\
\hline
\end{tabular}




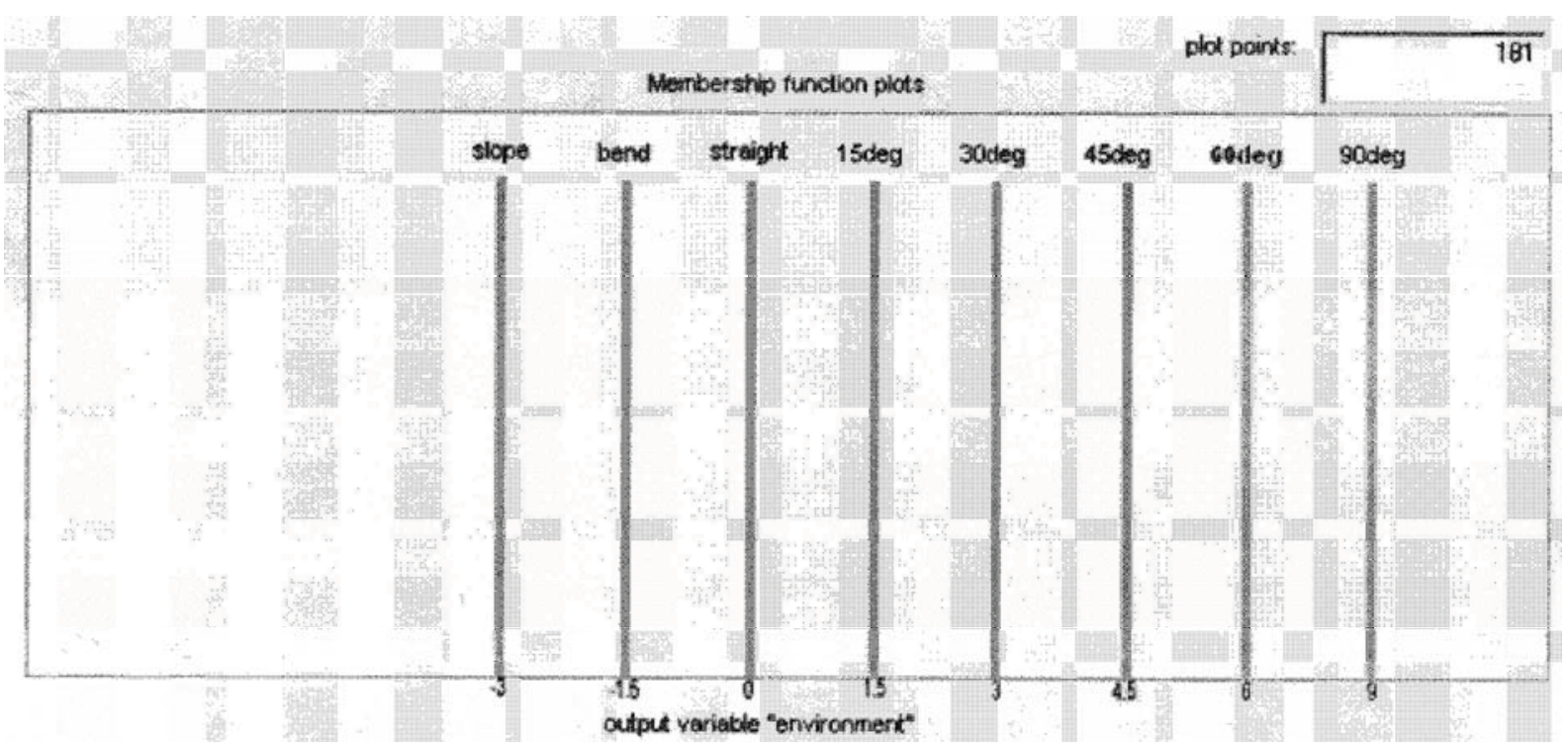

Fig. 6 Output types of the pipe recognition fuzzy inference subsystem

algorithm for the Takagi-Sugeno fuzzy model is the weighted average method, which produces the average value of all the consequents of the activated inference rules with respect to the total of membership degree [28]:

$$
\text { Defuzzified output }=\frac{\sum_{i=1}^{n} w_{i} \bar{z}_{i}}{\sum_{i=1}^{n} w_{i}}
$$

where

$$
\begin{aligned}
& n=\text { number of output sets } \\
& w_{i}=\text { degree of membership of the } i \text { th fuzzy rule } \\
& \bar{z}_{i}=\text { singleton value of output sets } i
\end{aligned}
$$

A set of 36 rules is formed to describe the environment. Some examples of the environment recognition rules are as follows:

(a) $R_{11}$ : if angle rate is zero and speed difference is not zero, then environment is straight

(b) $R_{21}$ : if angle rate is positively small and speed difference is positively medium, then environment is 30 deg bend

(c) $\mathscr{R}_{31}$ : if angle rate is negatively small and speed difference is negatively small, then environment is bend

(d) $\mathscr{R}_{41}$ : if angle rate is somewhat positively large and speed difference is positively large, then environment is $90 \mathrm{deg}$ bend

It can be seen from the fuzzy input variables in Fig. 5 that the triangular membership functions overlapping areas play an important part in the inference process. The overlapping areas in fuzzy membership function sets ensure that multiple rules will be executed, which means that any changes in the input states are detected and handled, thus keeping track of the environment changes. The sets are symmetrical about zero and are overlapped at approximately 25 per cent. However, 10 per cent over- lapping is applied at zero proximity in the angle rate variable, to provide a more accurate control at a slow driving speed. The percentage of overlapping satisfies the sum-to-one (or less) rule, which states that the sum of all points through the overlapping fuzzy membership functions should be equal to or less than one [29].

\subsection{Vehicle action fuzzy controller}

The cascaded hierarchical fuzzy evaluation has several advantages in the robotic system control ability. Firstly, it allows the reduction of the rules dimensionality, as mentioned previously, since there are a large number of input variables and membership functions in each variable. Secondly, it allows the use of a further state variable in conjunctive form with the pipe environment identifier outputs, thus producing a more accurate vehicle action inference. Thirdly, the method produces less discontinuity results since the crisp values of the inferred environment outputs are fuzzified for the vehicle action inference process.

The control of the vehicle motion within the pipe environment is highly dependent upon the pipe fittings, which can be distinguished using the vehicle speed and tilt measurements. The input variables (measured angle and environment type) to the vehicle action fuzzy subsystem are shown in Fig. 7. There are 11 membership functions for the measured climb angle input and 10 membership functions for the environment input. The latter membership function set is obtained using the pipe fittings recognition algorithm. Table 2 gives the linguistic descriptions for both sets of membership functions. There are six outputs from the action fuzzy controller as shown in Fig. 8.

There are a few ground rules on which the vehicle action if-then rules are based. The first one is the steady 


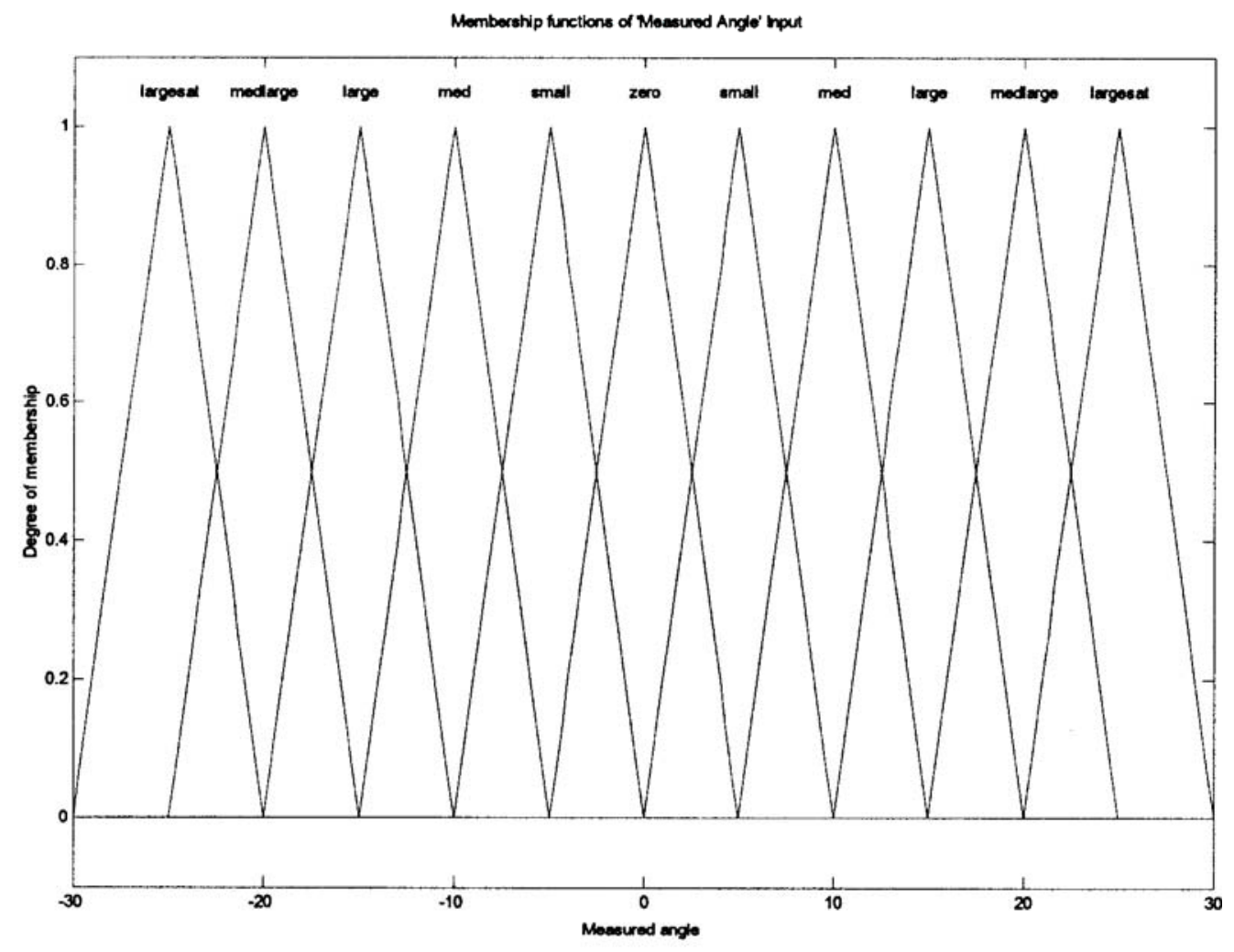

(a) Measured angle (deg)

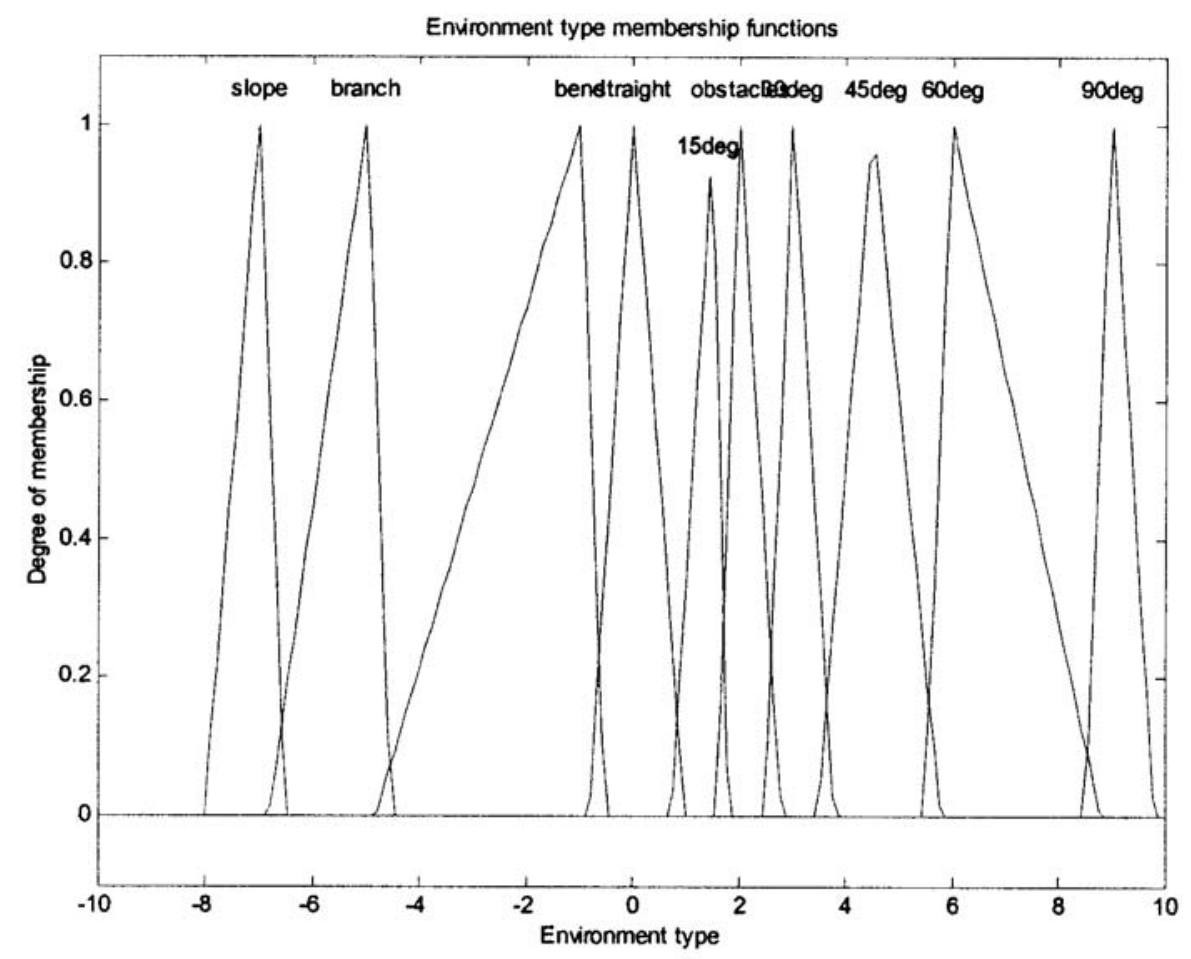

(b) Environment type (level)

Fig. 7 Input variable membership function sets for the vehicle action fuzzy controller 
Table 2 Linguistic description of (a) measure angle and (b) environment type input variables

\begin{tabular}{|c|c|c|c|}
\hline Label & Description & Label & Description \\
\hline \multicolumn{4}{|c|}{ (a) Measured angle membership set } \\
\hline Zero & Zero climb angle & Large & Large climb angle \\
\hline Small & Small climb angle & Med large & Very large climb angle \\
\hline Med & Medium climb angle & Large sat & Saturated climb angle \\
\hline \multicolumn{4}{|c|}{ (b) Environment membership set } \\
\hline Slope & Slope pipe section & $90 \mathrm{deg}$ & $90^{\circ}$ bend section \\
\hline Branch & Branch/junction section & $60 \mathrm{deg}$ & $60^{\circ}$ bend section \\
\hline Bend & Bend/curve section & $45 \mathrm{deg}$ & $45^{\circ}$ bend section \\
\hline Straight & Straight pipe section & $30 \mathrm{deg}$ & $30^{\circ}$ bend section \\
\hline Obstacle & Obstacle (not implemented) & $15 \mathrm{deg}$ & $15^{\circ}$ bend section \\
\hline
\end{tabular}

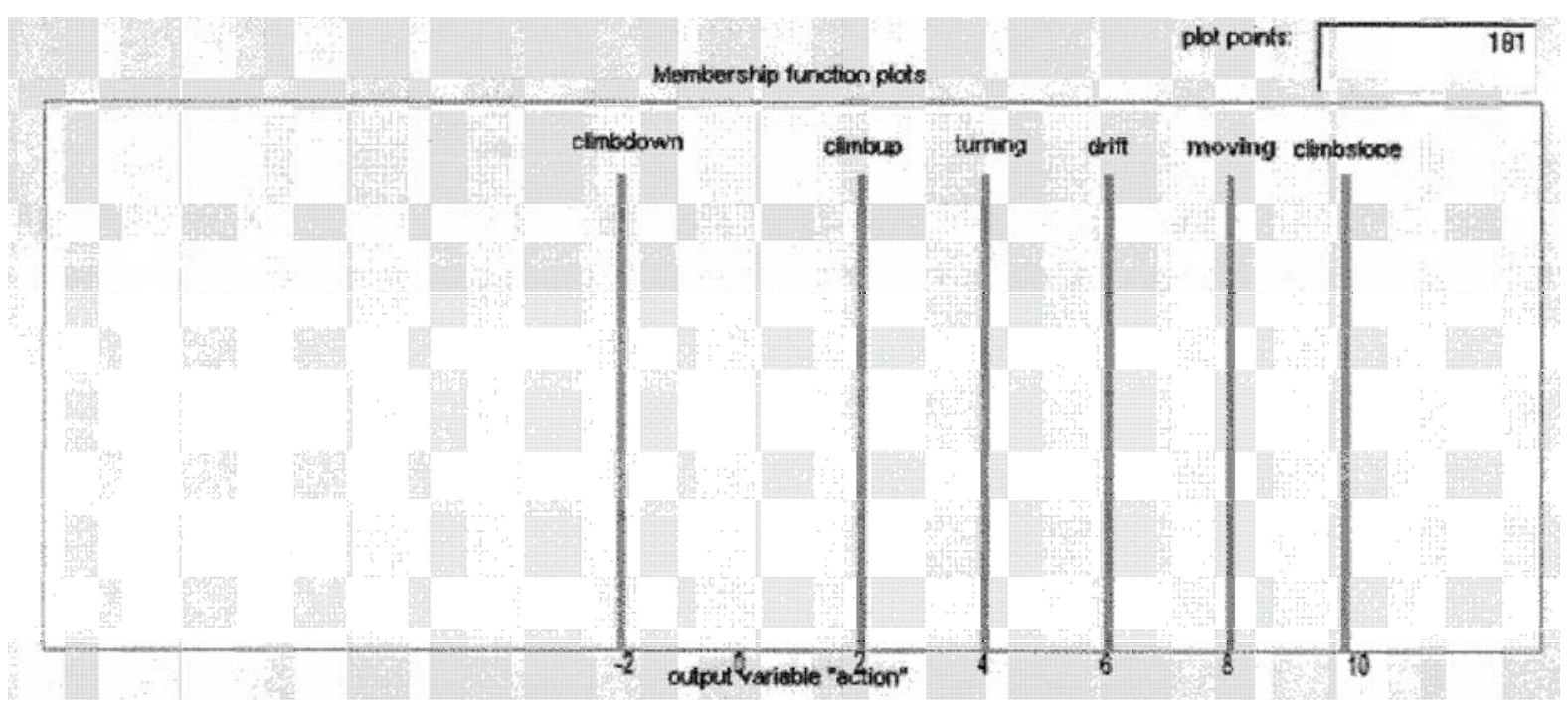

Fig. 8 Action type outputs of the fuzzy inference system

state principle, which states that no correction/adjustment action is taken when the state of the vehicle is in equilibrium. In the equilibrium state when the climb angle rate and the speed difference are zero, the speed of the motor is maintained at the input/desired value. The second principle governs the adjustments to vehicle actions and states that a change of actions is allowed only to negotiate the pipe environment (i.e. different pipe fittings). For this principle, comparisons between the vehicle actions and the perceived environment are carried out. If there is a change of climb angle, angle rate and speed difference, the fuzzy controller has to decide whether the behaviour is desirable by checking with the inferred environment. If it is allowed, the fuzzy controller produces an adjustment value in-line with the inputs. Similarly, when the action is not suited to the current environment, the fuzzy output reverses the vehicle action by adjusting the speed and climb angle back to the desired value. This is according to the 'changes not permitted' principle. Vehicle action corrections are achieved by adjusting the driving speed at each side to maintain position, restrict climbing angle or allow climbing.

Four of the example rules are given below. These are used to recognize actions such as drift, climbing, turning, etc., given the type of fittings such as a straight pipe or a bend section:

(a) $\Re_{21}$ : if measured angle is not zero, and environment is straight, then action is drifting

(b) $\mathscr{R}_{22}$ : if measured angle is small, and environment is $30 \mathrm{deg}$ bend, then action is climbing

(c) $R_{32}$ : if measured angle is small, and environment is bend, then action is climbing down

(d) $\mathscr{R}_{42}$ : if measured angle is large, and environment is $90 \mathrm{deg}$ bend, then action is turning

Example rule (a) allows the PID controller to maintain the vehicle's motion according to operator inputs since the vehicle is in a straight pipe and drifting action that causes swaying motion is not desired. Rules (b), (c) and (d) allow the fuzzy controller to produce the appropriate control signal to govern vehicle actions. Example rule (b) indicates that the vehicle is entering a $30^{\circ}$ bend. Example rule (c) prepares the vehicle to correct to its original orientation as it exits the bend. Example rule (d) gives a 'turning' control signal to the vehicle in a $90^{\circ}$ bend. 


\section{SIMULATION RESULTS}

To demonstrate the performance of the fuzzy navigation controller, a series of pipe fittings have been modelled in MATLAB, as illustrated in Fig. 9. A few virtual pipeline configurations, such as the ones shown in Fig. 10, have been built from the modelled pipe sections.

The navigation controller scheme for simulation is shown in Fig. 11. Two user inputs, i.e. input forward/ reverse speed and climb angle, can be set. The 'input angle' is used by the operator to move the vehicle up the side of the pipe wall to avoid obstacles and pipe takeoffs. It is not used for negotiating bends as this is done automatically without the user's input. A detailed fuzzy subsystems configuration of the fuzzy inference system is shown in Fig. 11 b.

\subsection{Pipe environment recognition simulations}

For the simulation tests, the maximum range of tilt angle is set to $\pm 30^{\circ}$. This is to prevent the vehicle from top-
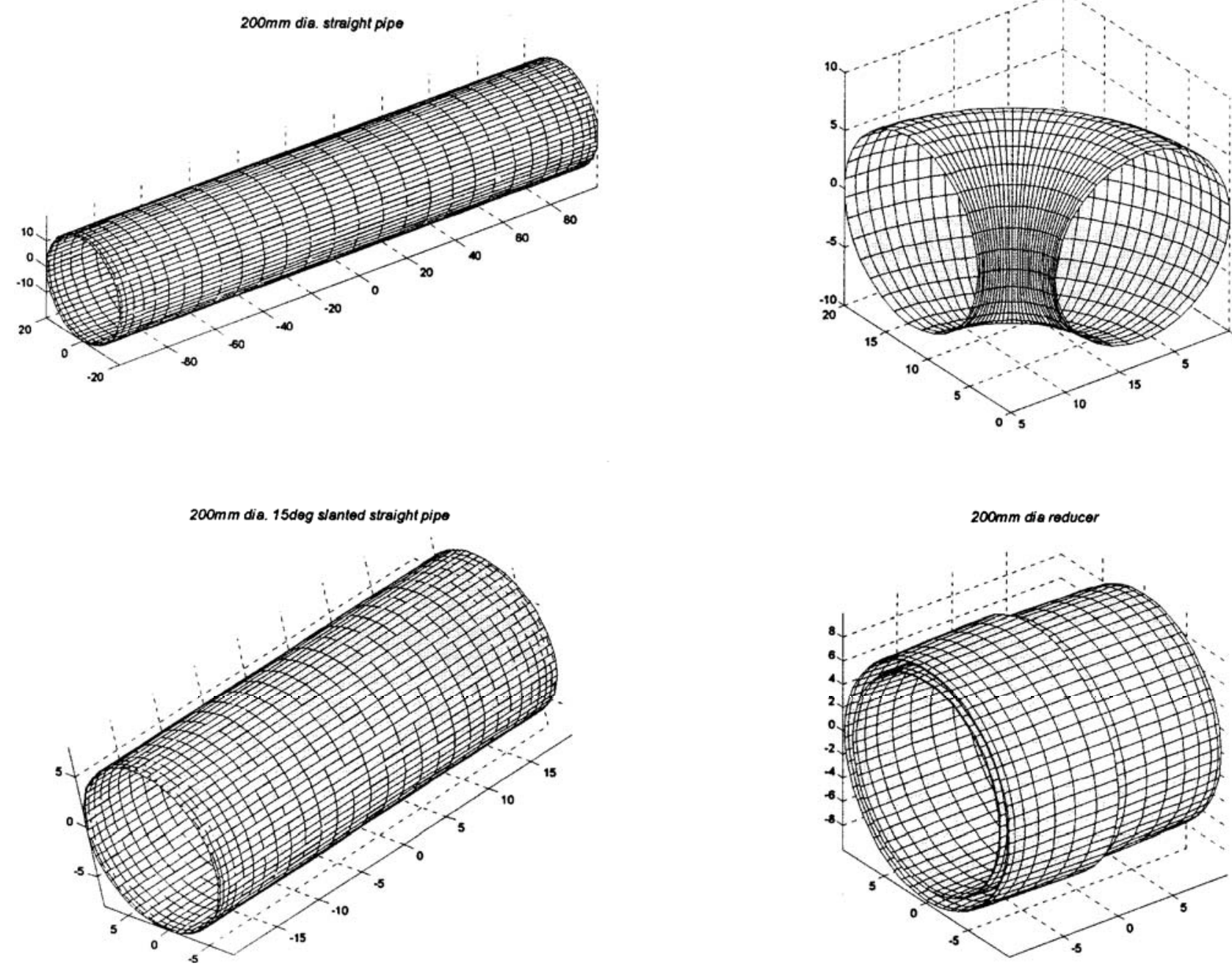

Fig. 9 Simulated pipe fittings pieces mum speed of the vehicle. Figures 12 and 13 show two case studies of a recognition process for pipe fittings. In the first case, the vehicle is driven through a $200 \mathrm{~mm}$ diameter straight pipe section, followed by a $15^{\circ}$ bend, a $45^{\circ}$ bend, a $60^{\circ}$ bend and a $90^{\circ}$ bend. The pipe fittings recognition fuzzy inference output in Fig. 12 shows a distinctive and consistent recognition of all the pipe fittings. The control surface in Fig. 12 illustrates the detected transitions of the various pipe sections across the valid range of speed and angle rate. The fuzzy inference outputs for a $45^{\circ}$ bend and a $60^{\circ}$ bend vary slightly from the desired values at a lower vehicle speed. These slight variations may be due to sensitivity and damping settings of the tilt sensor, which in turn will influence the interpretations of the fuzzy controller. The tilt sensor sensing is based on a spirit level principle. Nevertheless, the sensor effect has been dampened by the triangular membership functions and the inference outputs are well within the correct range for each type of fitting.

Figure 13 shows the simulation results of the vehicle driving through a straight pipe, followed by a $15^{\circ}$

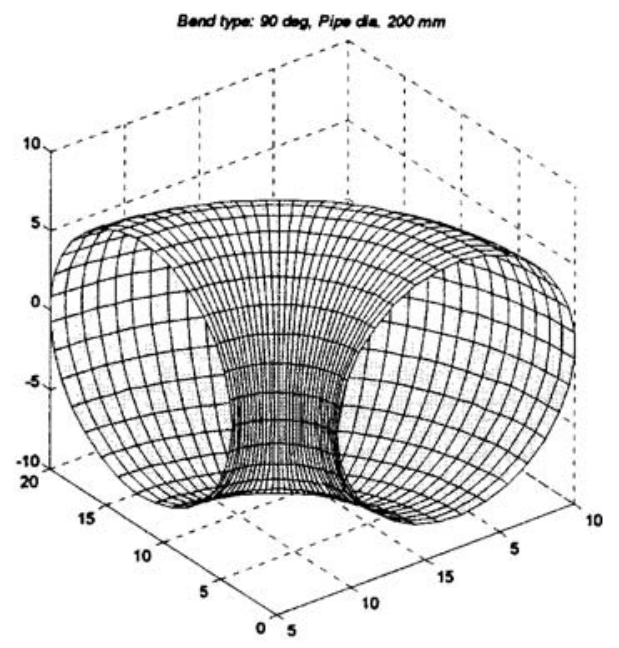




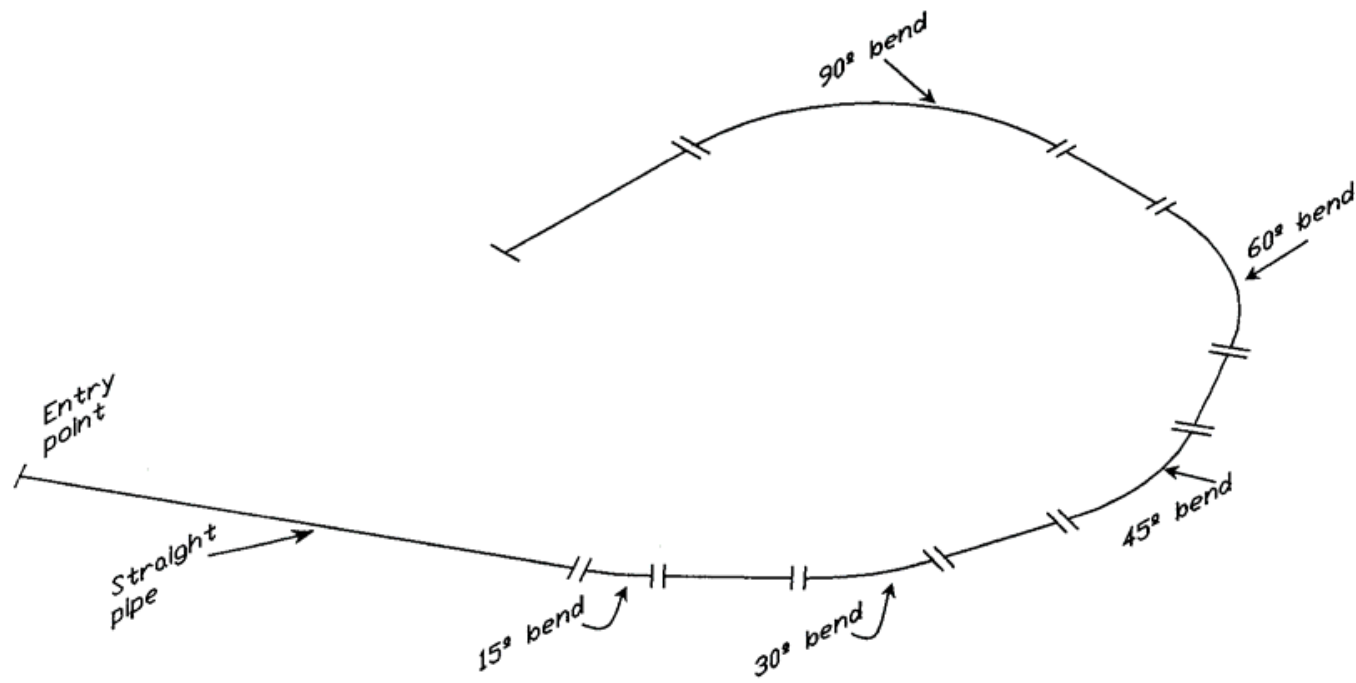

(a) Example of a virtual pipe configuration (straight pipe section, followed by a $15^{\circ}$, a $30^{\circ}$, a $45^{\circ}$, a $60^{\circ}$ and a $90^{\circ}$ bend)

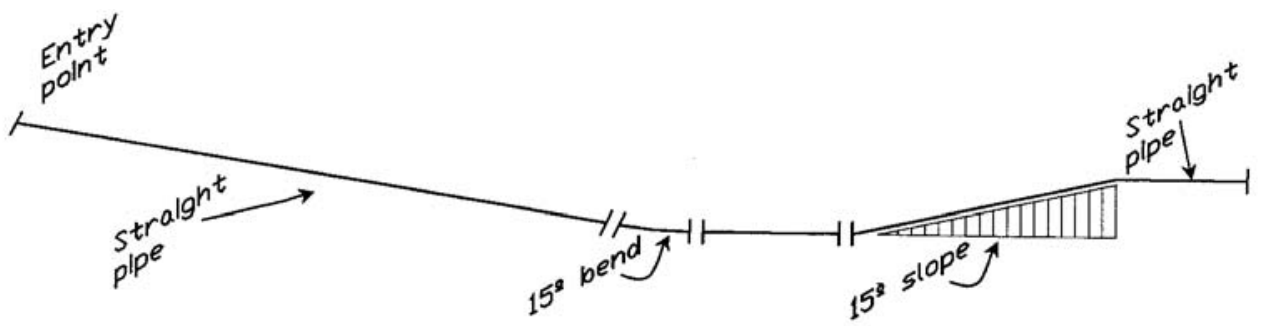

(b) Example of a virtual pipe configuration (straight pipe section, followed by a $15^{\circ}$ and a15 $5^{\circ}$ vertical inclined straight pipe)

Fig. 10 Simulated pipeline configurations for the navigation controller simulation

horizontal bend and then a $15^{\circ}$ vertical slope section. Similar to the previous case, the transition from the straight pipe to the $15^{\circ}$ horizontal bend is indicated by a positive gradient and the $15^{\circ}$ vertical slope is indicated by a change in gradient, in this case a negative gradient. Both upward and downward slopes give the same results. The gradient denotes the sensitivity of the inference process to the variations of the input values.

\subsection{Robotic system motion control simulations}

Figure 14 shows the vehicle action responses as it drives through various pipe fittings. The control surface contour in Fig. 14 shows a smooth transition between different types of vehicle action. For example, the surface contour for the vehicle 'turning' action in a $60^{\circ}$ bend shows a smooth transition of the action type, starting from the 'climb up' action as the vehicle enters the bend to 'turning' while the vehicle is in the bend. Another smooth action type output that can be observed is the 'climb slope' action for when the vehicle goes up or down a sloped pipe. However, the gradual gradient of the 'climb slope' action indicates a slightly longer response time. The correction action signal in the block diagram of Fig. 11 is computed from the 'action' type output and the vehicle speed to produce the correction in left and right speed levels.

It should be noted that the degree of overlapping of membership functions in the environment input to the action controller (as shown in Fig. 7b) plays an important part in the way that they provide the continuity associated with the segmentation and classification of an otherwise discrete input. Therefore, it can be said that the overlap property is not an artefact of fuzzy reasoning but reflects the actual nature of the fuzzy sets underlying the variable's domain.

\section{EXPERIMENTAL RESULTS AND DISCUSSION}

Given these encouraging simulation results, it is then feasible to implement the hierarchical fuzzy navigation 


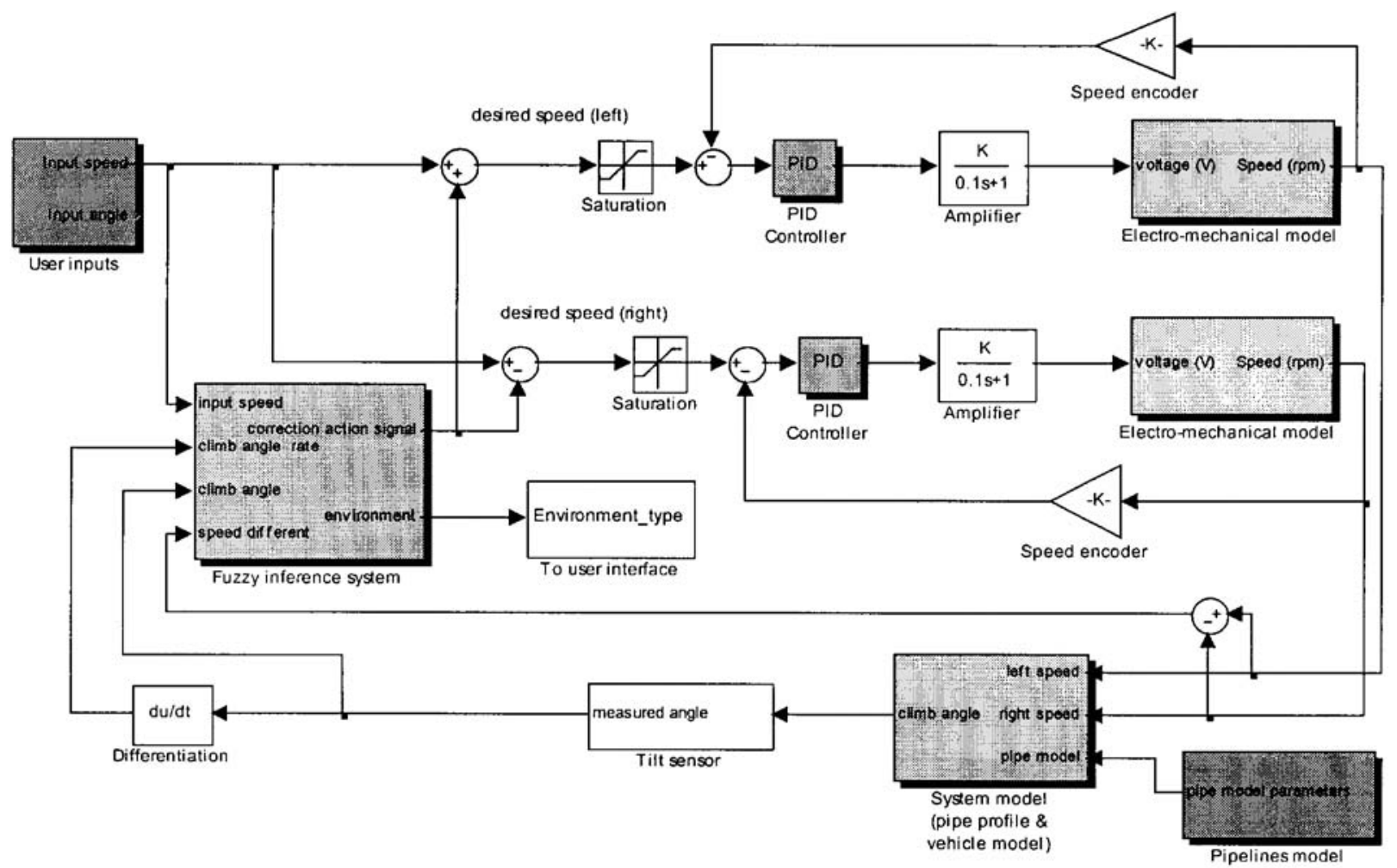

(a) Robotic vehicle navigation controller

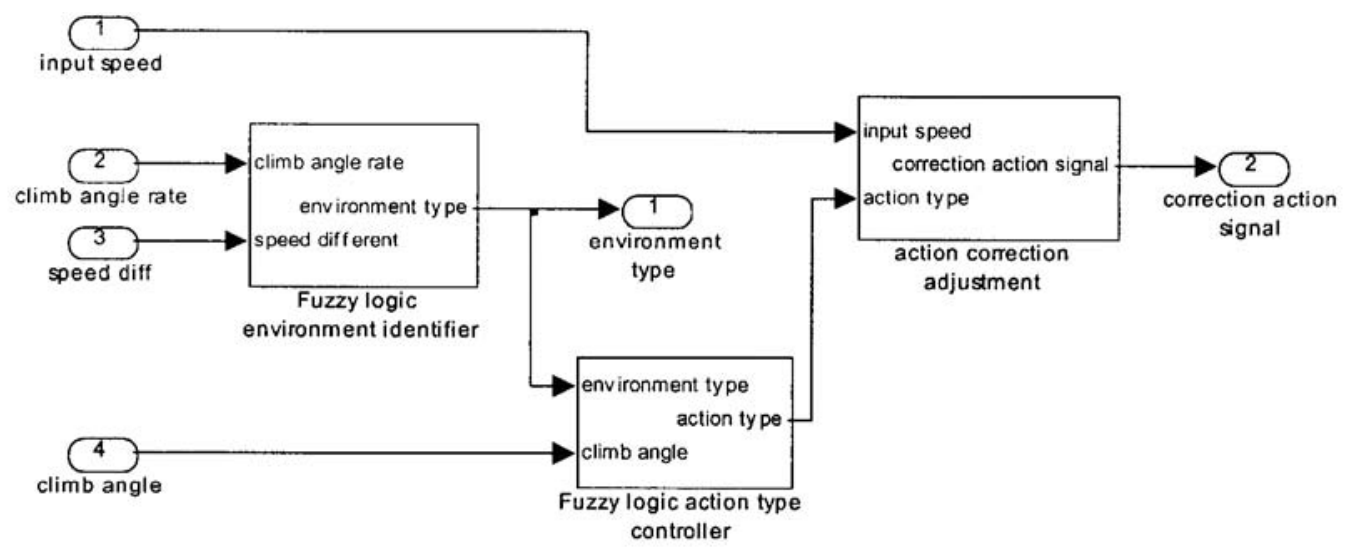

(b) Subsystems of the fuzzy inference system block

Fig. 11 Navigation controller simulation diagrams

controller for practical experiments. The fuzzy controller is implemented into the layer of an operating graphical user interface (GUI) on a separate computer. The GUI allows the operator to the input desired speed and climb angle of the vehicle. The software performs fuzzy inference processes in the background while the sensor data are being updated. The PID controller is implemented on-board the robotic vehicle for motion control, i.e. to minimize speed and climbing angle errors. A microcontroller board (using a PIC16F876 processor) was designed and used to implement the PID control algorithm.
In the experiments, the robotic vehicle is required to carry out sensor-based navigation in the pipe configuration, as shown in Fig. 15, i.e. to navigate:

(a) from location A to location B and

(b) from location $\mathrm{B}$ to location $\mathrm{C}$.

Location A to B consists of a $200 \mathrm{~mm}$ diameter straight pipe, followed by a $90^{\circ}$ bend. From location $\mathrm{B}$ to $\mathrm{C}$ is a $200 \mathrm{~mm}$ trunk line with a side take off in the midsection and an expander/reducer placed at the end. Additional experiments have been carried out involving

Proc. Instn Mech. Engrs Vol. 217 Part I: J. Systems and Control Engineering 
Pipe environment type for pipe environment fuzzy identifier output

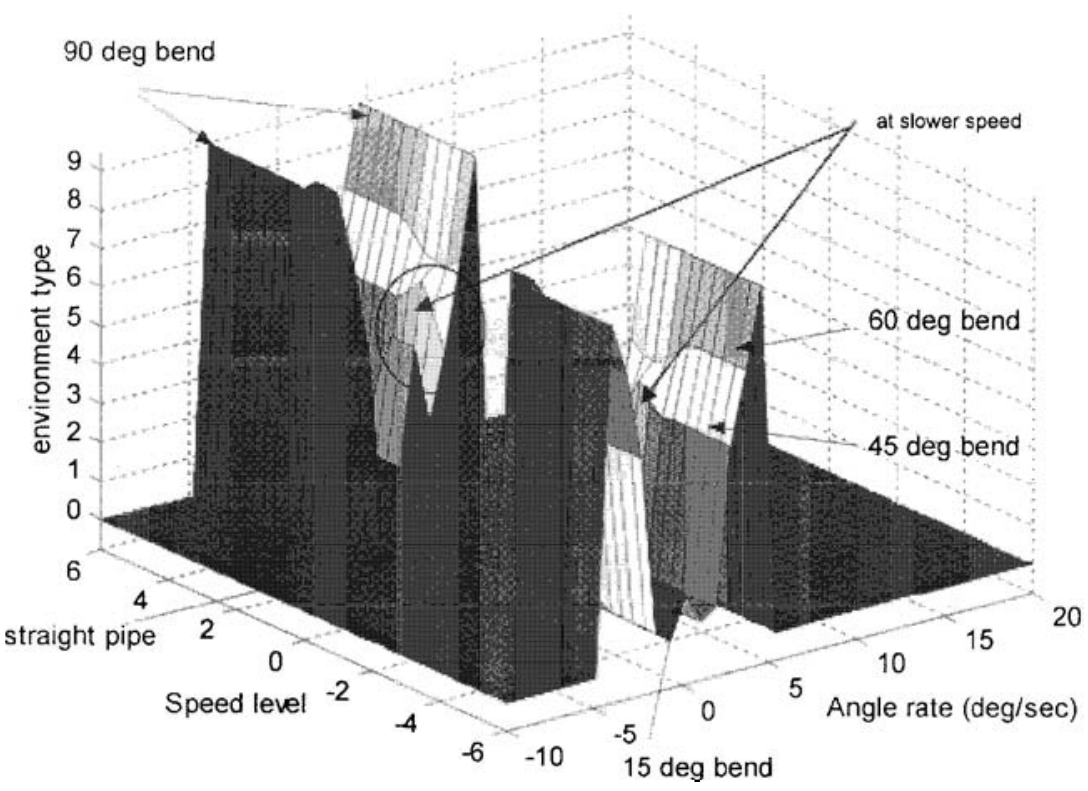

Fig. 12 Pipe recognition fuzzy identifier simulation results (200 $\mathrm{mm}$ diameter pipe section, followed by a $15^{\circ}, 45^{\circ}, 60^{\circ}$ and $90^{\circ}$ bend )

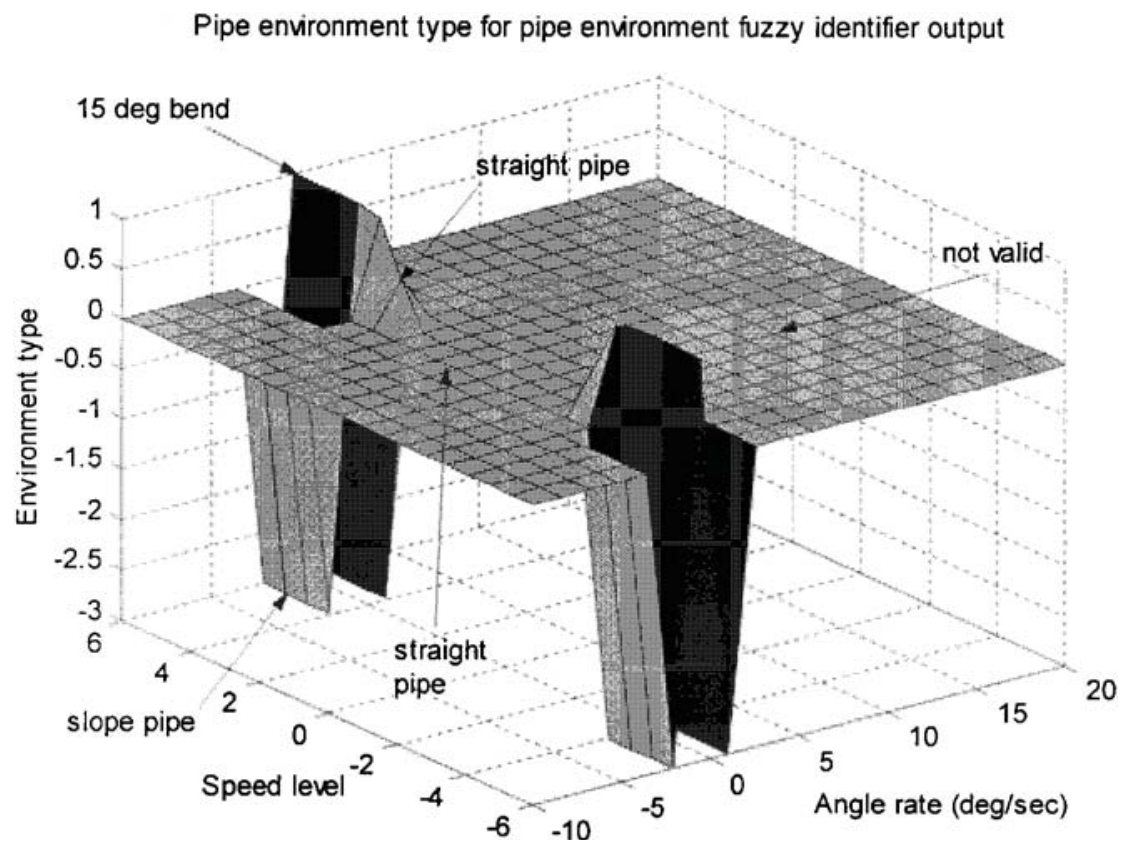

Fig. 13 Pipe recognition fuzzy identifier simulation results $(200 \mathrm{~mm}$ diameter pipe section, followed by a $15^{\circ}$ bend and a $15^{\circ}$ vertical slope pipe)

the navigation of the vehicle in large-diameter straight pipes. A $250 \mathrm{~mm}$ pipe has been used in both horizontal and $15^{\circ}$ inclined arrangements. Similar to the simulation experiments, the vehicle motion (speed and tilt angle) is observed and the environment type identifier outputs and the action type inference outputs are obtained using the cascaded hierarchical fuzzy system configuration shown in Fig. 4 and described in section 4.1.
Figures 16 and 19 to 21 show the real time navigation inference outputs for both the environment and vehicle action types obtained from the experiments. The evaluation of the robotic vehicle system is based on the following functionalities:

(a) the interpretation of pipe environment from sensory data; 


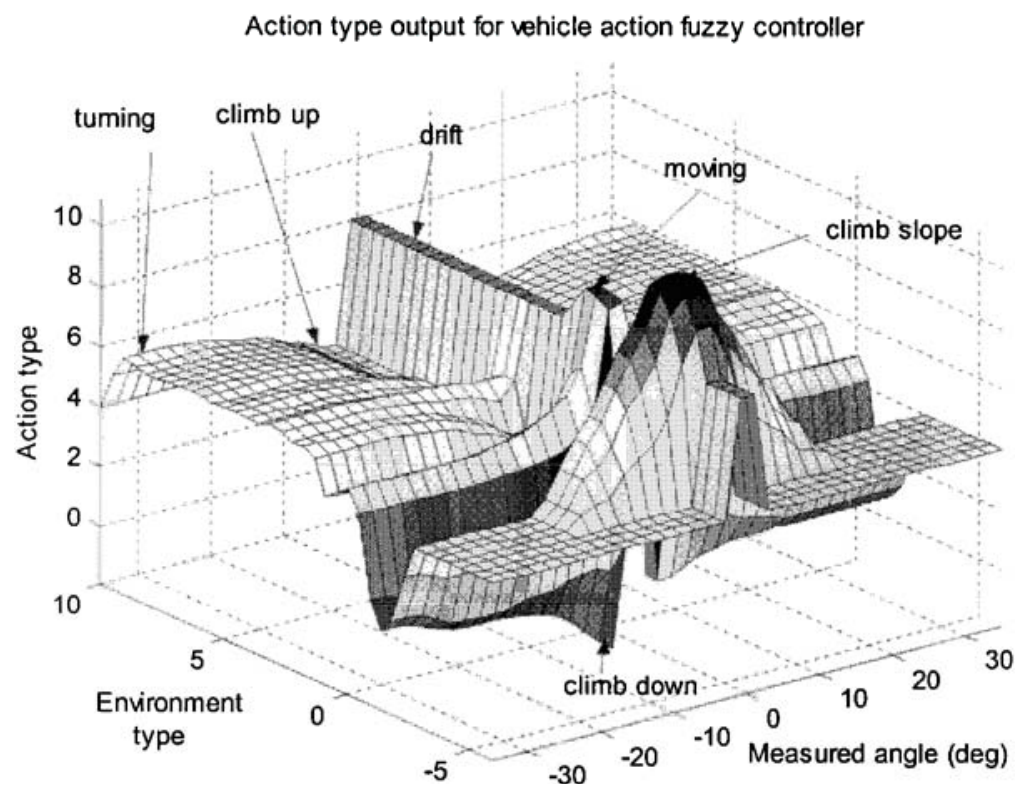

Fig. 14 Control surface of simulated action fuzzy outputs

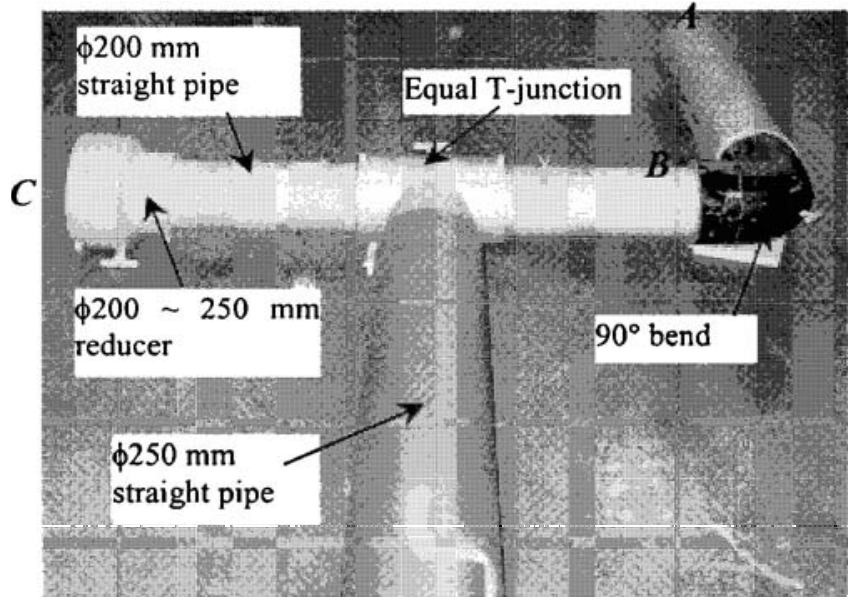

Fig. 15 Pipeline configuration for experiments

(b) the control of vehicle motion in the pipe environment;

(c) the accuracy of the environment interpretation and vehicle motion control.

In Fig. 16, the vehicle starts at $\mathrm{A}$ and moves along a straight pipe section before entering the bend section at $\mathrm{P} 1$. The $90^{\circ}$ bend is detected less than $1 \mathrm{~s}$ after the vehicle enters the bend. This is equivalent to approximately $6 \mathrm{~cm}$ into the bend and before the turning point. The environment fuzzy identifier interpreted the entry of the bend as a gradual change of bend types, as can be observed from the rising slope in the figure. As defined previously in section 4.3 and shown in Fig. 8, action type 8 is shown for the vehicle moving in the straight pipe section and action type 2 is shown for the vehicle entering the bend. A gradual change in action type is observed during the entry of the bend. The action type fuzzy controller interprets such action type levels and provides the vehicle controller with appropriate speed difference adjustments. As soon as the environment fuzzy controller determines that the pipe section is a $90^{\circ}$ bend, the action control signal reaches the 'turning' steady state value. The gradual change is important because if the vehicle is given the 'turning' control signal too soon, it is likely to get stuck in the bend section. The action type fuzzy inference outputs during the exiting of the vehicle from the bend are the opposite reasoning as for the entrance. The effectiveness of the navigation fuzzy controller is demonstrated in Figs 17 and 18. Figure 17 shows the vehicle entering a $90^{\circ}$ bend section from the zero angle position with a slight climb angle and Fig. 18 shows the vehicle in the middle of the bend section with a larger climb angle.

In the second experiment the robotic system is driven in the extended straight pipe section from B to C. Along the way, three distinctive responses from the navigation controller have been observed as shown in Fig. 19. Close examination of the pipe configuration shows that they refer to the connecting flanges on the pipe $\mathrm{T}$-junction section and the reducer section, which produce $15 \mathrm{~mm}$ steps at the connections. The environment type identifier regarded the steps as an approximation of a $15^{\circ}$ bend and the action controller responded as 'climb up'. It is noted also that the environment type identifier output regards the reducer section as a straight pipe section, and the possible corresponding vehicle actions are 'moving' and 'drift'. In this case, the action type fuzzy controller interprets these actions as 'moving', i.e. no adjustment in speed, since the climb angle and angle rate are either very small or zero.

Figure 20 shows the environment and action types for the robotic vehicle driven in a $15^{\circ}$ inclined $250 \mathrm{~mm}$ diameter straight pipe. It can be seen that the inclined pipe section is not immediately detected. At the entrance of 
From location A-B

(straight -90 deg bend)

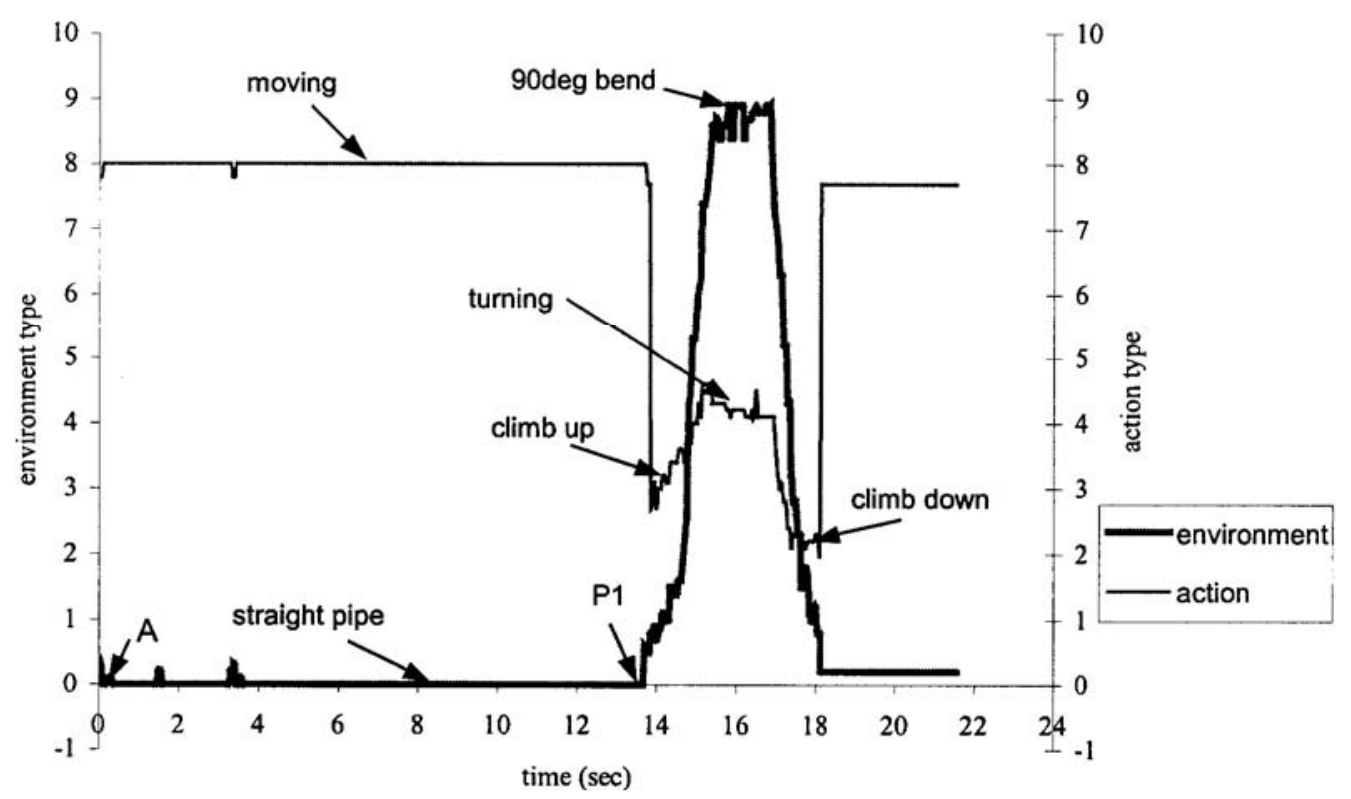

Fig. 16 Experimental results for location A-B

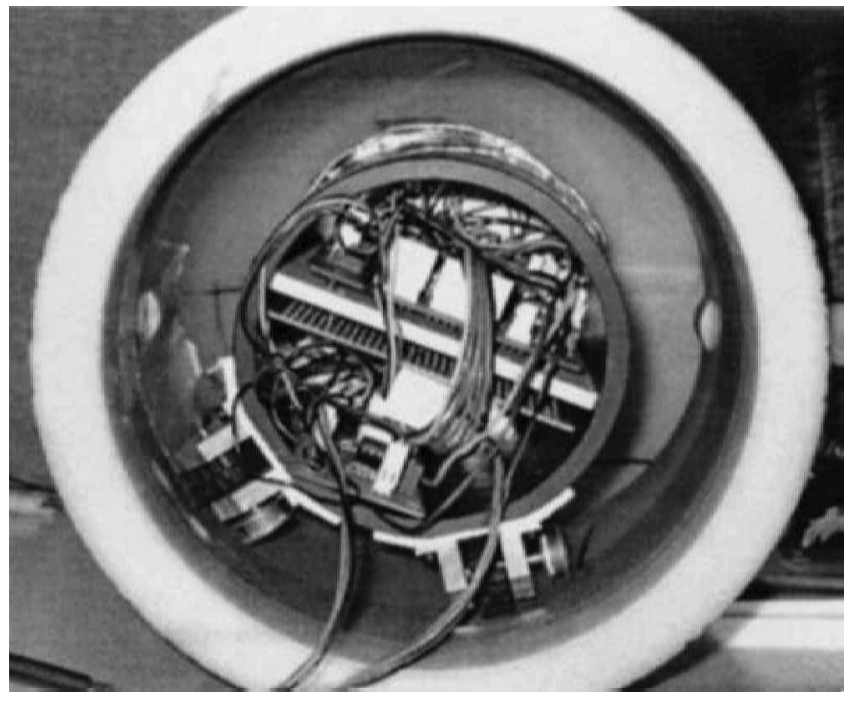

Fig. 17 Robotic vehicle entering a $90^{\circ}$ bend

the pipe section, the environment type identifier produced a defuzzified value of a straight pipe section, and the action type is given as a 'moving' signal. As the robotic vehicle moved further, the navigation controller subsequently produced the correct combination of environment and action types for a straight pipe. Hence, the left and right speed settings of the vehicle are not changed by the fuzzy inference system since the 'climb slope' action type produces a zero speed adjustment.

In the final experiment, the robotic vehicle was driven at approximately $14 \mathrm{~cm} / \mathrm{s}$, which is twice as fast as in the rest of the experiments. The aims were to observe the actual vehicle behaviour as well as the overall response

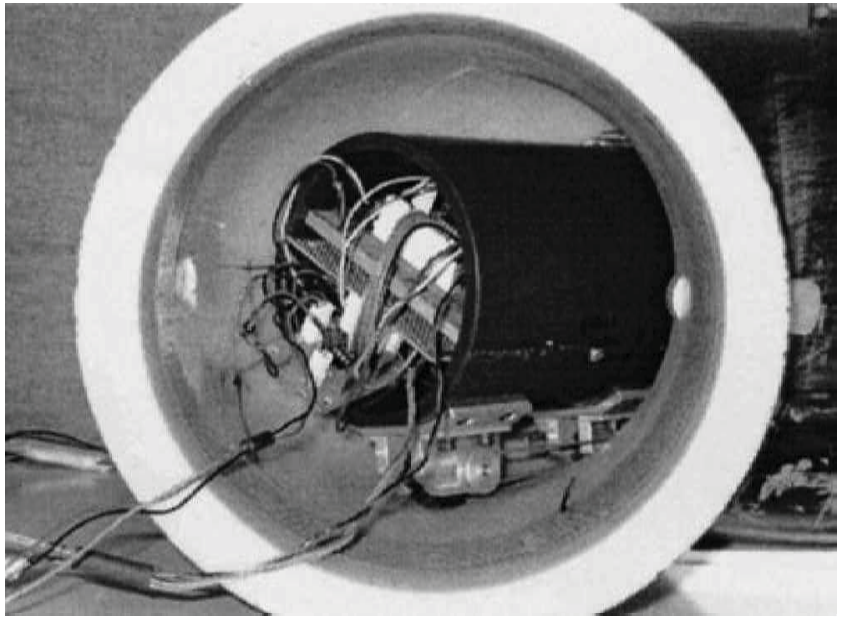

Fig. 18 Robotic vehicle in a $90^{\circ}$ bend

of the fuzzy navigation system. A larger diameter pipe was used, which reduces the constrained space. There is more 'drift' in this type of environment, as appropriately detected by the action type fuzzy controller shown in Fig. 21. The variations of the vehicle states were not large enough to cause the environment type identifier to detect other types of environment other than a 'straight' pipe section.

\section{CONCLUSION}

As can be seen from the fuzzy navigation responses as well as from the robotic vehicle actions in simulation and experimental tests, the fuzzy navigation system has 


\section{From location B-C}

(straight - T-junction - straight - reducer)

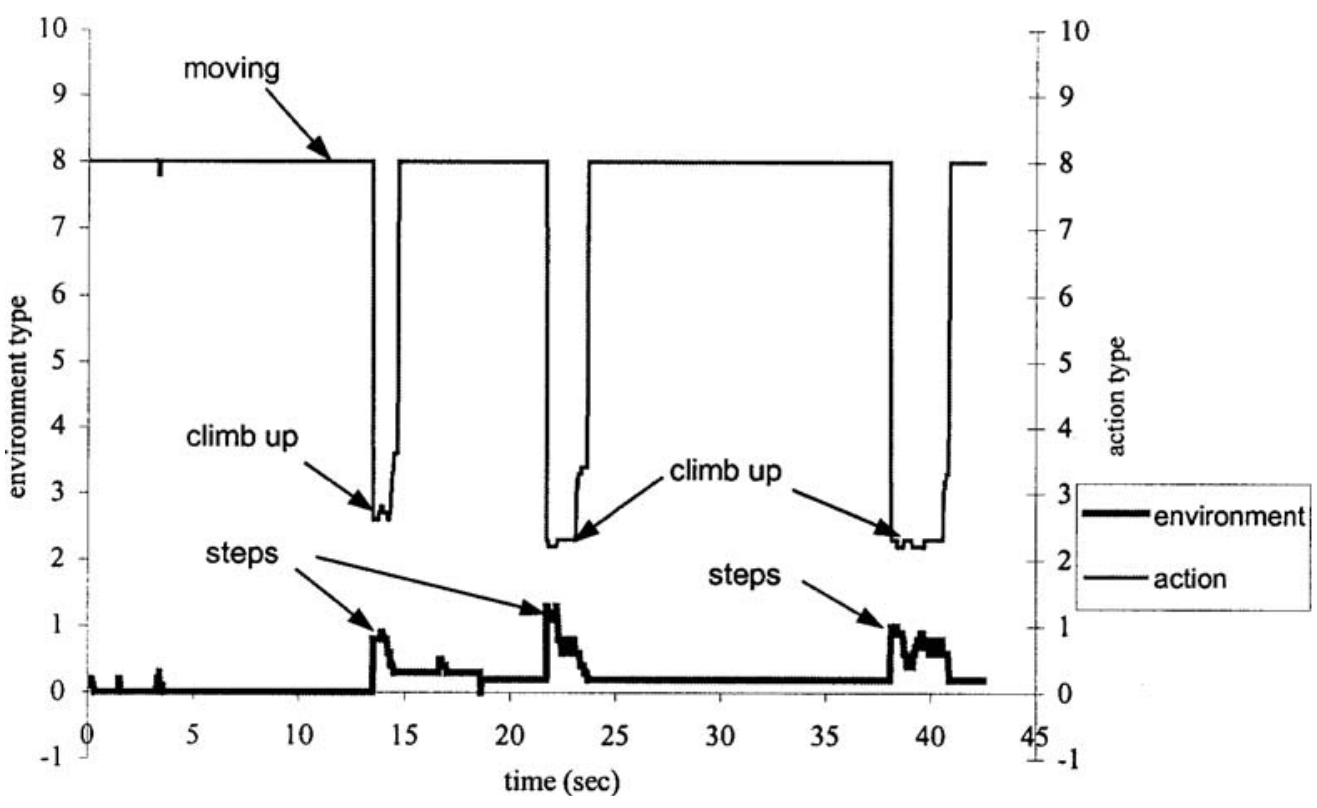

Fig. 19 Experimental results for location B-C

$250 \mathrm{~mm}$ dia. straight pipe, $15 \mathrm{deg}$ inclination

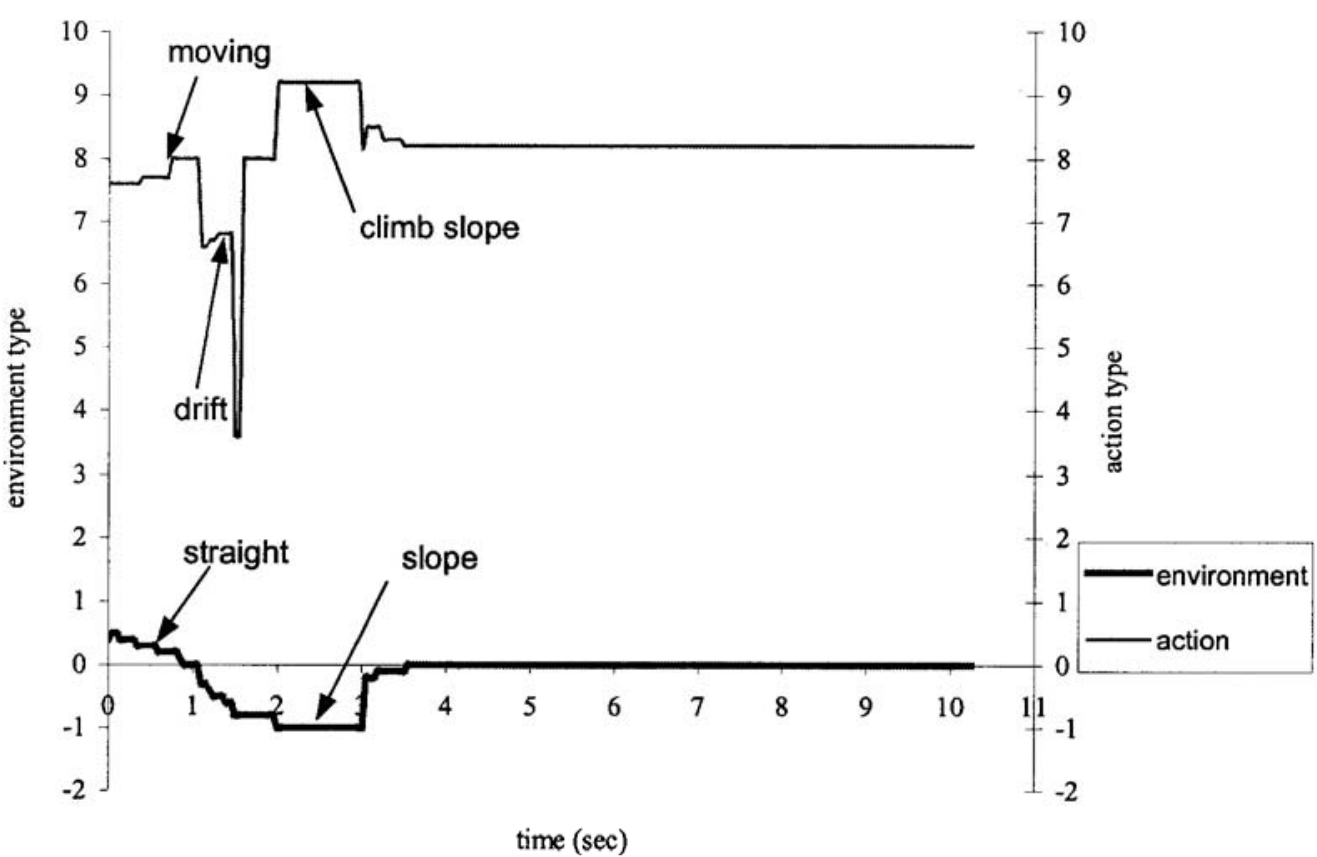

Fig. 20 Experimental results for a $15^{\circ}$ inclined straight pipe

been proven to work well. The navigation control strategy, as a whole, can be categorized as a reactive control system with a 'sense-interpret-act' control cycle. This means that the robotic system motion is adjusted through a knowledge of the environment. This methodology provides a more rapid and flexible response, which is attainable through a hybrid of traditional and intelligent methods of control. The usefulness of this control strategy is that it does not maintain or depend on environment models such as visual data. Creating and processing a representation of the environment in such a way is a time consuming and error-prone process. Instead, as shown in the paper, the robotic vehicle system reacts directly to the environment stimuli presented by a tilt sensor and optical encoders.

A fuzzy model configuration, termed the 'cascaded Proc. Instn Mech. Engrs Vol. 217 Part I: J. Systems and Control Engineering 
$250 \mathrm{~mm}$ dia. straight pipe, driving speed at $14 \mathrm{~cm} / \mathrm{s}$

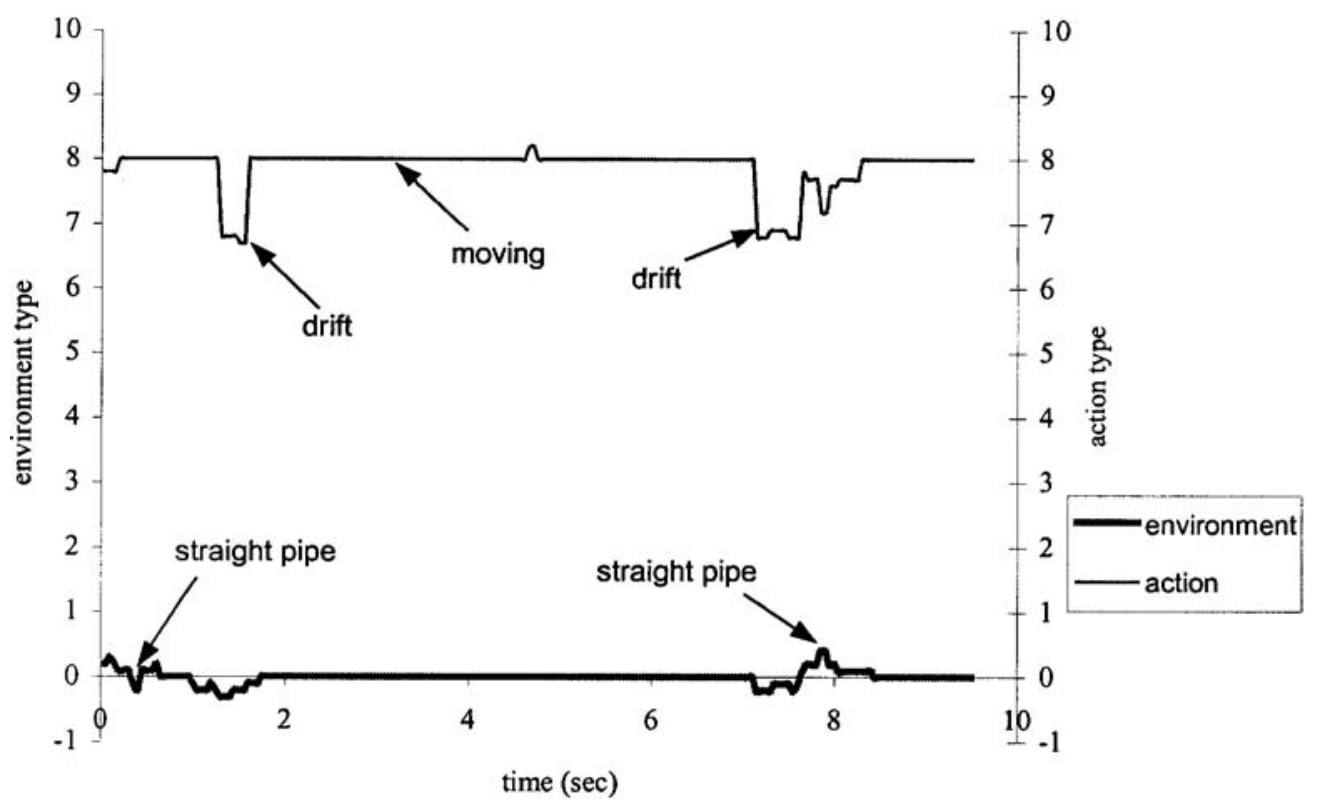

Fig. 21 Environmental and action type outputs for a faster driving speed

hierarchical configuration' is used to reduce the 'curse of fuzzy rule dimensionality' due to the MIMO system, as well as to provide an accurate pipe environment dependent vehicle action. The two-stage controller performs better than the composite fuzzy navigation controller. The results of sections 5 and 6 have shown in detail how the navigation controller operates successfully both in simulations and in practice.

The self-navigation solution is believed to be able to remedy a few limitations of current gas pipe operation robotic tools in the near future, such as by allowing the deployment of multiple robotic systems and increasing their reliability by using a simpler mechanical drive.

\section{REFERENCES}

1 Cohen, J. H. Robotic Applications for Gas Distribution Industry, 1998, p. 187 (Maurer Engineering Inc., Houston, Texas).

2 Hirose, S., Ohno, H., Mitsui, T. and Suyama, K. Design of in-pipe inspection vehicles for $\varnothing 25, \varnothing 50, \varnothing 150$ pipes. In IEEE International Conference on Robotics and Automation, Detroit, Michigan, 1999.

3 Suzumori, K., Miyagawa, T., Kimura, M. and Hasegawa, Y. Micro inspection robot for 1 -in pipes. IEEE/ASME Trans. Mechatronics, 1999, 4(3), 286-291.

4 Gamble, G. B. and Wiesmann, R. M. Tethered Mouse System for Inspection of Gas Distribution Mains, 1996, p. 143 (Foster-Miller Inc., Waltham, Massachusetts).

5 Hapstack, M. and Talarek T. R. Pipe crawlers: versatile adaptations for real applications. In 14th Robots Conference, Detroit, Michigan, 1990.

6 Jezequel, P. Mobile robot for pipe inspection and maintenance (mobile robots II). Proc. SPIE, 1987, 852, 282-287.
7 Bradshaw, A. and Counsell, J. M. A knowledge based mechatronics approach to controller design. In UKACC International Conference on CONTROL '98, Swansea, 1998 (Institute of Electrical Engineers).

8 Normey-Rico, J. E., Gomez-Ortega, A. I. and Camacho, E. F. Mobile robot path tracking using a robust PID controller. Control Engng Practice, 2001, 9, 1209-1214.

9 Tang, J., Watanabe, K., Nakamura, M. and Koga, S. Fuzzy Gaussian neural network controller and its application to the control at a mobile robot. Nippon Kikai Gakkai Ronbunshi, 1993, 59(564), 2290-2297.

10 Saffiotti, A. Fuzzy Logic in the Autonomous Mobile Robot FLAKEY, 1992; http://iridia.uld.ac.be/saffiotti/flakeybib. html.

11 Song, K. and Tai, J. Fuzzy navigation of a mobile robot. In Proceedings of the 1992 IEEE International Conference on Intelligent Robots and Systems, Rayleigh, USA, 1992.

12 Owen, C. and Nehmzow, U. Landmark based navigation for a mobile robot. In Simulation of Adaptive Behaviour, 1998 (MIT Press, Cambridge, Massachusetts).

13 Fukuda, T., Ito, S., Arai, F. and Yokoyama, Y. Navigation system based on ceiling landmark recognition for autonomous mobile robot. In Proceedings of the 1995 IEEE/RSJ International Conference on Robots and Systems, Pittsburgh, Pennsylvania, 1995, Vol. 2, pp. $150-155$.

14 Maeyama, S., Ohya, A. and Yuta, S. Non-stop Outdoor Navigation of a Mobile Robot. In Proceedings of the InternationalConference on Intelligent Robots and Systems (IROS), 1996, Vol. 1, pp. 130-135.

15 Becker, C., Salas, J., Tokusei, K. and Latombe, J.-C. Reliable navigation using landmarks. In IEEE International Conference on Robotics and Automation, Nagoya, Japan, 1995.

16 Rencken, W. D. Autonomous sonar navigation in indoor, unknown and unstructured environments. In International 
Conference on Intelligent Robots and Systems (IROS '94), Munich, Germany, 1994.

17 Luo, R. C. and Pan, T. J. An intelligent path planning system for robot navigation in an unknown environment. SPIE Mobile Robots IV, 1989, 1195, 316-326.

18 Ong, J. K., Kerr, D. and Bouazza-Marouf, K. Design of a semi-autonomous modular robotic vehicle for gas pipeline inspection. Proc. Instn Mech. Engrs, Part I: J. Systems and Control Engineering, 2003, 217(I2), 109-122.

19 Ong, J. K., Kerr, D. and Bouazza-Marouf, K. In-pipe multirobot system: modular co-operative semi-autonomous robotic units. In International Gas Research Conference (IGRC 2001), Amsterdam, Netherlands, 2001.

20 Ong, J. K., Kerr, D. and Bouazza-Marouf, K. In-pipe robotic system. In 8th Mechatronics Forum International Conference (Mechatronics 2002), University of Twente, Netherlands, 2002.

21 Hertzberg, J., Christaller, T., Kirchner, F., Licht, U. and Rome, E. Sewer Robotics, 1998 (GMD-German National Research Centre for Information Technology, Sankt Augustin, Germany).

22 Fuzzy Logic Toolbox for Use with MATLAB, 2001 (The Mathworks Inc., Natick, Massachusetts).
23 Guven, M. K. and Passino, K. M. Avoiding exponential parameter growth in fuzzy systems. IEEE Trans. Fuzzy Systems, 2001, 9(1), 194-199.

24 Chiu, S. Method and software for extracting fuzzy classification rules by substructure clustering. In North American Fuzzy Information Processing Society, Initiative Soft Computing IEEE Neural Networks Council on System, Man and Cybernetics, 1996, pp. 19-22.

25 Halmagamuge, S. and Glesner, M. Neural networks in designing fuzzy systems for real world applications. Fuzzy Sets and Systems, 1994, 65(1), 1-12.

26 Ishibuschi, H., Nozaki, K., Yamamoto, N. and Tanaka, H. Selecting fuzzy if-then rules for classification using genetic algorithm. Fuzzy Sets and Systems, 1995, 3, 260-270.

27 Combs, W. and Andrews, J. E. Combinatorial rule explosion eliminated by a fuzzy rule configuration. IEEE Trans. Fuzzy Systems, 1998, 6, 1-11.

28 Ying, H. Fuzzy Control and Modeling: Analytical Foundations and Applications, 2000 (Institute of Electrical and Electronics Engineers Press, Piscataway, New Jersey).

29 Cox, E. The Fuzzy Systems Handbook-A Practitioner's Guide to Building, Using and Maintaining Fuzzy Systems, 2nd edition, 1999 (AP Professional, New York). 